\title{
Low-thrust propulsion in a coplanar circular restricted four body problem
}

\author{
Marta Ceccaroni · James Biggs
}

Received: date / Accepted: date

\begin{abstract}
This paper formulates a circular restricted four body problem (CRFBP), where the three primaries are set in the stable Lagrangian equilateral triangle configuration and the fourth body is massless. The analysis of this autonomous coplanar CRFBP is undertaken, which identifies eight natural equilibria; four of which are close to the smaller body, two stable and two unstable, when considering the primaries to be the Sun and two smaller bodies of the solar system. Following this, the model incorporates 'near term' low-thrust propulsion capabilities to generate surfaces of artificial equilibrium points close to the smaller primary, both in and out of the plane containing the celestial bodies. A stability analysis of these points is carried out and a stable subset of them is identified. Throughout the analysis the Sun-Jupiter-Asteroid-Spacecraft system is used, for conceivable masses of a hypothetical asteroid set at the libration point $L_{4}$. It is shown that eight bounded orbits exist, which can be maintained with a constant thrust less than $1.5 \times 10^{-4} N$ for a $1000 \mathrm{~kg}$ spacecraft. This illustrates that, by exploiting low-thrust technologies, it would be possible to maintain an observation point more than $66 \%$ closer to the asteroid than that of a stable natural equilibrium point. The analysis then focusses on a major Jupiter Trojan: the 624-Hektor asteroid. The thrust required to enable close asteroid observation is determined in the simplified CRFBP model. Finally, a numerical simulation of the real Sun-Jupiter-624 Hektor-Spacecraft is undertaken, which tests the validity of the stability analysis of the simplified model.
\end{abstract}

Keywords Restricted Problems · Stability · Periodic Orbits

Marta Ceccaroni

Advanced Space Concepts Laboratory

University of Strathclyde, Glasgow, UK

E-mail: marta.ceccaroni@strath.ac.uk

James Biggs

Advanced Space Concepts Laboratory

University of Strathclyde, Glasgow, UK

E-mail: james.biggs@strath.ac.uk 


\section{Introduction}

The general, spatial, four body problem is a complex 24 degree of freedom system. However, the system can be reduced by exploiting integrals of motion to obtain a 12 degree of freedom system but cannot be reduced further without loss of generality. Furthermore, it is well known that with four (or more) massive bodies there is no analytical stability criterion valid for all time (Steves et al., 1998b). Therefore, the analysis of such "non-restricted" models have mainly been limited to finding some stability criterion relative to a particular configuration. Examples include Milani and Nobili (1983), on a four body linear hierarchical ${ }^{1}$ system, and Roy et al. (1985), on fictitious coplanar four body systems with different distribution of masses and hierarchies, in which a numerical estimation of the duration of the stability for these systems is found. In order to derive stability properties over infinite time intervals, it is necessary to consider suitably simplified four body systems (Roy et al., 1985).

Examples of these "suitably simplified" models include autonomous configurations (given by the relative equilibrium solutions) such as the straight line (Multon, 1910), square, equilateral triangle and kite configurations (Multon (1900), Steves et al. (1998b), Pina and Lonngi (2009) and Sicardy (2011)), and non-autonomous models, such as the Caledonian Problem (namely the coplanar, initially circular, equal masses, four body problem), by Steves et al. (1998a). The latter is an important special case as, although it does not admit the Jacobi integral, it allows almost all the other conventional dynamical systems strategies to study the evolution and the stability of the trajectories (e.g. the identification of periodic orbits, the study of the linear stability (Hadjidemetriou, 1978) or the long term symplectic integration of the equations of motion). Another class of simplified cases fall into the category of the "restricted" FBPs where one of the four bodies is considered massless. A study of relative equilibria in restricted FBPs can be found in Simo' (1978). Furthermore, relative equilibrium solutions have been obtained in the special cases of equal mass symmetric configurations (e.g. the collinear symmetric CRFBP by Michalodimitrakis (1981)) or by using Hill's approximation (where the fourth body is assumed to be at a great distance and therefore its influence is reduced to a perturbing term, transforming the system into a perturbed restricted three body problem). Examples include Scheeres (1998) and Papadakis (2007), the bicircular (Cronin et al. (1964), Koon et al. (2008)), or the quasi-bicircular (Gomez et al., 2001) and the concentric circular models (Andreu, 2002). Among which papers (Gomez et al., 2001) and (Andreu, 2002) which use this model as an approximation to the Sun-Earth-Moon-Spacecraft system, highlight the lack of regions of "good stability properties" in the vicinity of the triangular lagrangian points of the smaller, perturbed system.

This paper investigates a particular restricted FBP, namely an autonomous (in a rotating frame) coplanar CRFBP, where the three massive bodies are set in the stable Lagrangian equilateral triangle configuration. This system, although dealing with the dynamics of three masses and a particle, is different from the previously mentioned restricted cases as it does not use Hill's approximation or the assumption of equal mass and symmetric configurations. There does exist a number of studies on restricted FBP set in such a configuration mainly used to model existing binary systems (see

\footnotetext{
1 a system is said to be hierarchical if at a given epoch it can be defined to exist as a clearly identified number of disturbed two body motions where the two bodies in a pair may be made up of masses, a mass and a center of mass or two centers of mass
} 
Kloppenborg et al. (2010), Melita et al. (2008), Van Hamme and Wilson (1986), and Schwarz et al. (2009)). Of particular relevance are the papers by Alvarez-Ramirez and Vidal (2009) and Baltagiannis and Papadakis (2011) where the latter uses a numerical approach to determine the number of equilibrium points and a linear stability analysis depending on the distribution of the masses.

The objective of this paper is to identify completely novel orbits both for mathematical interest as well as for potential future mission applications. Initially the natural evolution of this model is studied, which identifies eight natural equilibrium points; four of which are close to the asteroid. Following this, the system is perturbed by the inclusion of low-thrust propulsion (such as solar electric propulsion (SEP)) previously only considered in two and three-body restricted problems.

Space mission design for low-thrust spacecraft has been extensively investigated from the late 1990's. So far the two major types of low-thrust propulsion, which have been studied in this context, are solar sails and SEP, the latter considered in this paper. Research on this topic, at present, mainly focus on finding artificial equilibria as in Morimoto et al. (2007), McInnes et al. (1994) and Baig and McInnes (2008), on generating non-Keplerian periodic orbits using solar sails or low thrust, e.g. Morimoto et al. (2006), Waters and McInnes (2007), Baig and McInnes (2009) and McKay et al. (2011), on the systematic cataloguing of non-Keplerian orbits using SEP as in McKay et al. (2009), or on analyzing the stability properties of minimum-control artificial equilibrium points as in Morimoto et al. (2007) and Bombardelli and Pelaez (2011), all of which are set in restricted two or three body models.

Throughout the paper the low-thrust Sun-Jupiter-Asteroid-Spacecraft is analyzed, as a particular case, for a range of estimated masses of a hypothetical Asteroid set to be trapped at the Lagrangian point $L_{4}$. Surfaces of artificial equilibrium points are then identified and a stability analysis of them undertaken. This paper highlights the potential of this investigation for designing observation missions to the Jupiter Trojans. This swarm of asteroids has been recognized as a present target for space science missions, as understanding it may lead to clues to the origin and dynamical evolution of Jupiter itself (Shoemaker et al., 1988). Currently, the Trojan asteroids are completely unexplored and largely unknown and any visit by a spacecraft will revolutionize our current understanding of these bodies (Rivkin et al., 2009). Moreover, although moving on tadpole orbits around the $L_{4}$ and $L_{5}$ points of the Sun-Jupiter-Spacecraft system (see Marzari (2006)), these asteroids are often modeled as fixed in the equilateral triangle configuration, e.g. considering them as concentrated at $L_{4}$, (see Marzari et al. (2002), Steves et al. (1998b), Steves et al. (1998a)). This configuration is also considered as the Solar system example of the equilateral triangle relative equilibrium solution found by Lagrange (Dvorak et al. (2008) and Baltagiannis and Papadakis (2011).

However, as there are no important Trojan Asteroids in or close to the $L_{4}$ point of the Sun-Jupiter system, a numerical investigation was performed, in order to test the validity of the stability analysis of the model when the Asteroid is moved from the exact $L_{4}$ location to the actual librating orbit of some real Jupiter Trojan. There are a number of papers on the application of theoretical results to real systems (see Hou and Liu (2010), Marchal (2011), Pina and Lonngi (2009) and Hadjidemetriou (2008)). 


\section{The autonomous coplanar CRFBP}

In this paper an autonomous coplanar CRFBP is analyzed; it is the problem of determining the dynamics of a body $P_{S}$ that moves under the influence of the gravitational field generated by three massive bodies, $P_{j}$ of masses $m_{j}, j=1,2,3$ respectively (say $\left.m_{1} \geq m_{2} \geq m_{3}\right)$. It is called restricted as the body $P_{S}$ is assumed to have negligible mass $m_{S}=0$. Furthermore it is Coplanar and circular since all the massive bodies revolve in the same plane and with the same angular velocity $\omega$, following circular orbits around the barycenter of $P_{1}$ and $P_{2}$.

Finally, it is autonomous as, taking a rotating frame of reference $O_{x, y, z}$, where the $x / y$ plane contains the three bodies, centered in the center of rotation of the planets and revolving around the third axis with the same angular velocity $\omega$ of the bodies, all the primaries $P_{1}, P_{2}$ and $P_{3}$ will be fixed in the rotating frame.

Moreover, in this paper, the primaries are set in the Lagrangian equilateral triangle configuration where the position of the third primary $P_{3}$ corresponds to one of the triangular Lagrangian points, such that the three massive bodies form an equilateral triangle, as in Ambrosetti and Prodi (1993).

This configuration is well known to be stable if the masses of the three planets satisfy the condition $\frac{m_{1} m_{2}+m_{1} m_{3}+m_{2} m_{3}}{\left(m_{1}+m_{2}+m_{3}\right)^{2}}<\frac{1}{27}$ (see Gascheau (1843), Routh (1875), Erdi et al. (2009) and Schwarz et al. (2009)); this condition is equivalent to $27\left(m_{1} m_{2}+\right.$ $\left.m_{1} m_{3}+m_{2} m_{3}\right)-\left(m_{1}+m_{2}+m_{3}\right)^{2}<0$.

As we assumed $m_{3} \leq m_{2}$ and the left term of this inequality is monotonically increasing in $m_{3}, \forall m_{3} \in\left(0, m_{2}\right)$, such left term is maximized for $m_{3}=m_{2}$, thus: $27\left(m_{1} m_{2}+m_{1} m_{3}+m_{2} m_{3}\right)-\left(m_{1}+m_{2}+m_{3}\right)^{2}<27\left(2 m_{1} m_{2}+m_{2}^{2}\right)-\left(m_{1}+2 m_{2}\right)^{2}$ $\forall m_{1}, m_{2}$.

Therefore the stability condition becomes: $-m_{1}^{2}+50 m_{1} m_{2}+23 m_{2}^{2}<0$ (more restrictive then the previous), that rearranged is: $\frac{m_{1}}{m_{2}}>25+18 \sqrt{2}$. In addition, the mass of the Asteroid is taken to be small enough not to influence the motion of the main primaries $P_{1}$ and $P_{2}$ (i.e. the center of rotation of the system remains in the barycenter of the two main bodies).

Scaled units of measure for mass and distance are used, normalized with the sum of the masses of $P_{1}$ and $P_{2}$ and their distance respectively, while the gravitational constant $\mathcal{G}$ and the rotational velocity $\omega$ of the system are set to 1 . In nondimensional units let $\mu, 1-\mu$ and $\epsilon$ be the scaled mass of $P_{2}, P_{1}$ and $P_{3}$ respectively, where $\mu=\frac{m_{2}}{m_{1}+m_{2}}$, $1-\mu=\frac{m_{1}}{m_{1}+m_{2}}, \epsilon=\frac{m_{3}}{m_{1}+m_{2}}$ and $m_{3} \ll m_{1}, m_{2}$.

As the system of reference has its origin in the barycenter $O$ and it rotates with the same angular velocity of $P_{1}$ and $P_{2}$, these two planets will be fixed and, without loss of generality, we can consider their positions to be $P_{1}=(-\mu, 0,0), P_{2}=(1-\mu, 0,0)$. This implies that, in order to form an equilateral triangle with them, the position of the third primary has to be: $P_{3}=\left(L_{x}, L_{y}, 0\right)=\left(\frac{1}{2}-\mu, \frac{\sqrt{3}}{2}, 0\right)$. The system just described is shown in Figure 1.

The dynamics of the massless spacecraft, whose state vector is expressed in nondimensional cartesian coordinates $\mathrm{x}=\left[x, y, z, v_{x}, v_{y}, v_{z}\right]^{T}$, is described by the system:

$$
\left\{\begin{array}{l}
\ddot{x}=2 \dot{y}-\frac{\partial \Omega}{\partial x} \\
\ddot{y}=-2 \dot{x}-\frac{\partial \Omega}{\partial y} \\
\ddot{z}=-\frac{\partial \Omega}{\partial z}
\end{array}\right.
$$




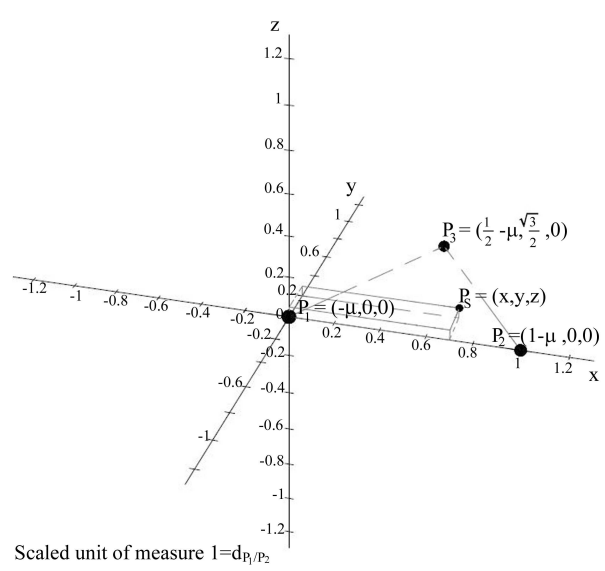

Fig. 1 The autonomous coplanar CRFBP; in a rotating frame of reference, the planets are fixed and form an equilateral triangle

where the augmented or effective potential and the distances of the spacecraft from each of the three primaries are respectively:

$$
\begin{aligned}
& \Omega=-\frac{x^{2}+y^{2}}{2}-\frac{(1-\mu)}{r_{1}}-\frac{\mu}{r_{2}}-\frac{\epsilon}{r_{3}}, \\
& r_{1}=\sqrt{(x+\mu)^{2}+y^{2}+z^{2}}, \\
& r_{2}=\sqrt{(x+\mu-1)^{2}+y^{2}+z^{2}}, \\
& r_{3}=\sqrt{\left(x-L_{x}\right)^{2}+\left(y-L_{y}\right)^{2}+z^{2}},
\end{aligned}
$$

and $\frac{\partial \Omega}{\partial x}, \frac{\partial \Omega}{\partial y}$ and $\frac{\partial \Omega}{\partial z}$ are the partial derivative of $\Omega$ with respect to $x, y$ and $z$, while the "dot" symbolizes differentiation with respect to time.

Note that if $\epsilon \rightarrow 0$, system (1) degenerates to the classical CR3BP, while if both $\epsilon \rightarrow 0$ and $\mu \rightarrow 0$ it becomes a R2BP.

In order to find the equilibrium points of the system, the velocities $\dot{x}, \dot{y}, \dot{z}$ and the accelerations $\ddot{x}, \ddot{y}, \ddot{z}$ are set to be zero in (1) obtaining:

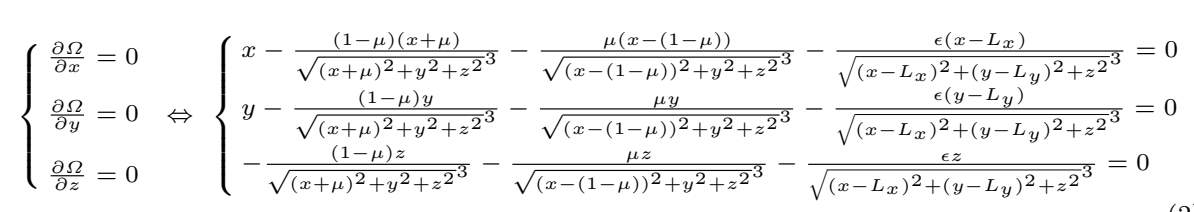

As we are in the stable Lagrangian configuration such system admits eight solutions (see Baltagiannis and Papadakis (2011)), the equilibrium points $M_{j}, j=1, \ldots, 8$, defined at the intersections of the three surfaces described by its equations, see Appendix A.

Figures 2 and 3 show the behavior of the three surfaces which satisfy system (2) in the vicinity of the third primary; in this region such surfaces form four equilibrium points $M_{4}, M_{5}, M_{6}$ and $M_{7}$ on which this paper is focussed. The former shows the three surfaces in three dimensions, while the latter represents them sectioned by the $z=0$ plane, part a), and intersected with this plane, part b).

In particular, in these Figures, the continuous, light, meshed surfaces represent the solution of the first equation $\frac{\partial \Omega}{\partial x}=0$ of system (2), the dashed, dark, meshed surfaces 
show the solution of $\frac{\partial \Omega}{\partial y}=0$, while the solution of the third equation is the plane $z=0$, plotted in the Figures as well. As the third equation is satisfied by the plane $z=0$, all the equilibria are bounded to stay on this plane, which, equivalently, can be seen as the degeneration of system (2) into a two dimensional system for $z=0$ (see Ceccaroni and Biggs (2010)).

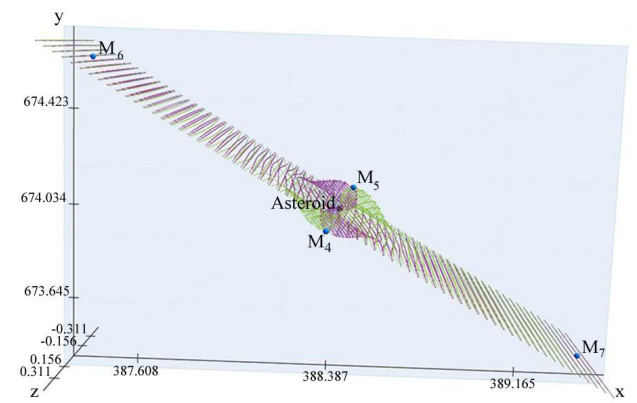

Unit of measure $1=10^{6} \mathrm{~km}$

Fig. 2 The four equilibria close to the asteroid; spatial view

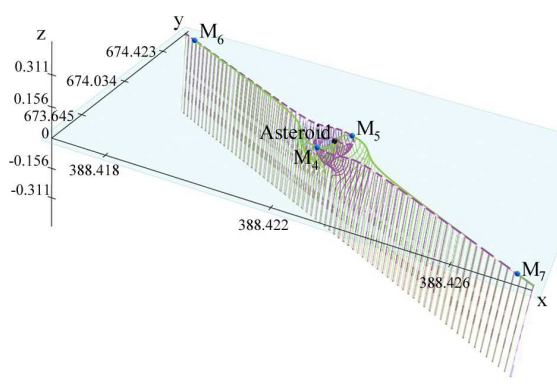

Unit of measure $1=10^{6} \mathrm{~km}$

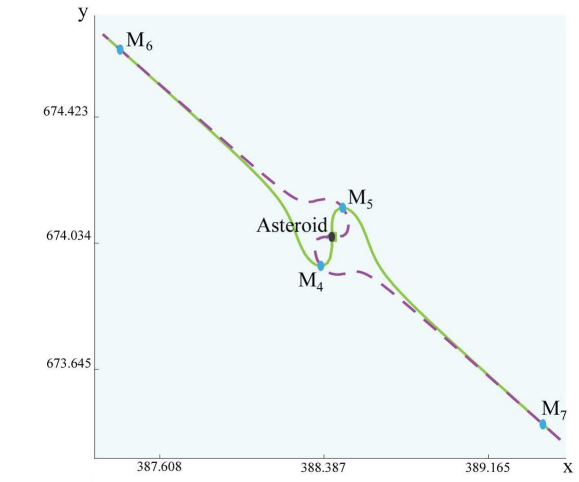

Unit of measure $1=10^{6} \mathrm{~km}$

Fig. 3 The four equilibria close to the asteroid; a) $z=0$ section of the spatial view; b) Intersection with the plane $z=0$

The qualitative dynamics close to the asteroid do not change for different masses of the asteroid $\epsilon \in(0, \mu)$, in that, there are always four equilibrium points configured approximately at the same angle relative to the asteroid. However, quantitatively, as the mass increases, the equilibrium points are displaced further from the asteroid, 
as shown in the Figure 4 a) and b) where the first shows the behavior of the four equilibrium points as the mass of the asteroid increases while the second is focussed just on $M_{4}$ and $M_{5}$.

Thus, we would conclude that the only assumptions on the masses of our system should be the stability condition, which, in nondimensional units, becomes: $\mu<$ $\frac{(13-9 \sqrt{2})}{14}$ and that the mass of the asteroid has to be small enough such that it does not affect the motion of the other two Primaries.

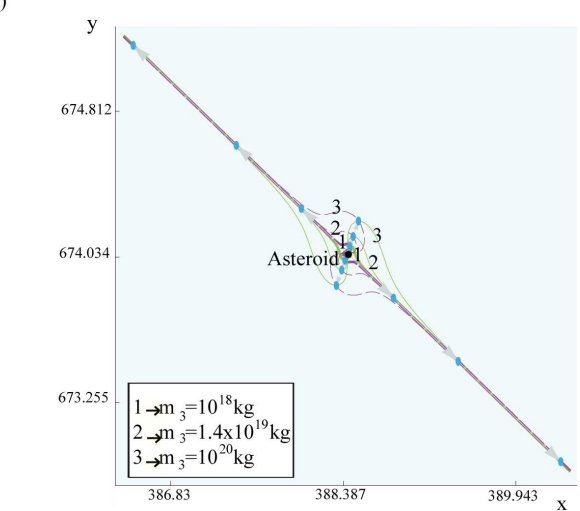

Unit of measure $1=10^{6} \mathrm{~km}$

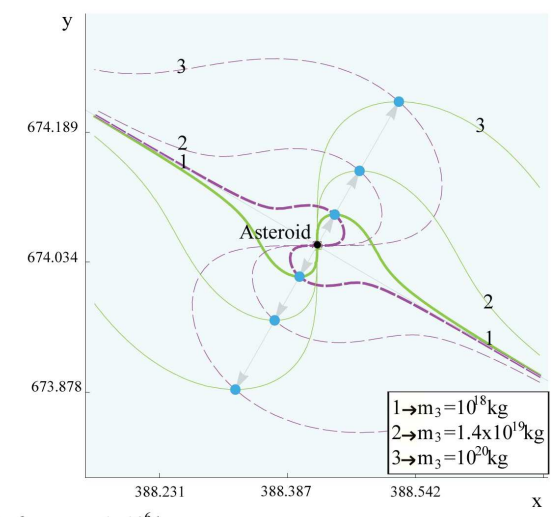

Fig. 4 Equilibrium Points; a) $\forall \epsilon \in(0, \mu)$ the two lines intersects four times in the region near the asteroid; b) Zoomed image

\section{Stability analysis}

Hereafter, for simplicity of notation, $\left(x_{e}, y_{e}, z_{e}\right)$ will indicate a generic equilibrium solution of system (2); moreover, for convenience, a translation to a generic equilibrium point $\left(x_{e}, y_{e}, z_{e}\right)$ is performed:

$$
\left\{\begin{array}{l}
x^{\prime}=x-x_{e} \\
y^{\prime}=y-y_{e} \\
z^{\prime}=z-z_{e}
\end{array}\right.
$$

but, to simplify notation, we will ignore the indices above $x^{\prime}, y^{\prime}, z^{\prime}$.

Then we linearize the motion close to this generic point $\left(x_{e}, y_{e}, z_{e}\right)$, obtaining

$$
\left\{\begin{array}{l}
\dot{x}=v_{x} \\
\dot{y}=v_{y} \\
\dot{z}=v_{z} \\
\dot{v_{x}}=2 v_{y}+\alpha x+\chi y+\delta z \\
\dot{v_{y}}=-2 v_{x}+\chi x+\beta y+\phi z \\
\dot{v_{z}}=\delta x+\phi y+\gamma z
\end{array}\right.
$$


with

$$
\begin{aligned}
& \alpha=1+\frac{(1-\mu)\left[2\left(x_{E}+\mu\right)^{2}-y_{E}^{2}-z_{E}{ }^{2}\right]}{\sqrt{\left(x_{E}+\mu\right)^{2}+y_{E}^{2}+z_{E}^{2}}}+\frac{\mu\left[2\left(x_{E}+\mu-1\right)^{2}-y_{E}{ }^{2}-z_{E}{ }^{2}\right]}{\sqrt{\left(x_{E}+\mu-1\right)^{2}+y_{E}^{2}+z_{E}^{2}}}+\frac{\epsilon\left[2\left(x_{E}-L_{x}\right)^{2}-\left(y_{E}-L_{y}\right)^{2}-z_{E}{ }^{2}\right]}{\sqrt{\left(x_{E}-L_{x}\right)^{2}+\left(y_{E}-L_{y}\right)^{2}+z_{E}^{2}}}, \\
& \beta=1+\frac{(1-\mu)\left[-\left(x_{E}+\mu\right)^{2}+2 y_{E}^{2}-z_{E}^{2}\right]}{\sqrt{\left(x_{E}+\mu\right)^{2}+y_{E}^{2}+z_{E}^{2}}}+\frac{\mu\left[-\left(x_{E}+\mu-1\right)^{2}+2 y_{E}^{2}-z_{E}^{2}\right]}{\sqrt{\left(x_{E}+\mu-1\right)^{2}+y_{E}^{2}+z_{E}^{2}}}+\frac{\epsilon\left[-\left(x_{E}-L_{x}\right)^{2}+2\left(y_{E}-L_{y}\right)^{2}-z_{E}^{2}\right]}{\sqrt{\left(x_{E}-L_{x}\right)^{2}+\left(y_{E}-L_{y}\right)^{2}+z_{E}^{2}}}, \\
& \gamma=\frac{(1-\mu)\left[-\left(x_{E}+\mu\right)^{2}-y_{E}^{2}+2 z_{E}^{2}\right]}{\sqrt{\left(x_{E}+\mu\right)^{2}+y_{E}^{2}+z_{E}^{2}}}+\frac{\mu\left[-\left(x_{E}+\mu-1\right)^{2}-y_{E}{ }^{2}+2 z_{E}^{2}\right]}{\sqrt{\left(x_{E}+\mu-1\right)^{2}+y_{E}^{2}+z_{E}^{2}}}+\frac{\epsilon\left[-\left(x_{E}-L_{x}\right)^{2}-\left(y_{E}-L_{y}\right)^{2}+2 z_{E}^{2}\right]}{\sqrt{\left(x_{E}-L_{x}\right)^{2}+\left(y_{E}-L_{y}\right)^{2}+z_{E}^{2}}}{ }^{E}, \\
& \chi=3\left\{\frac{(1-\mu)\left[\left(x_{E}+\mu\right) y_{E}\right]}{\sqrt{\left(x_{E}+\mu\right)^{2}+y_{E}^{2}+z_{E}^{2}} 5}+\frac{\mu\left[\left(x_{E}+\mu-1\right) y_{E}\right]}{\sqrt{\left(x_{E}+\mu-1\right)^{2}+y_{E}^{2}+z_{E}^{2}}} 5+\frac{\epsilon\left[\left(x_{E}-L_{x}\right)\left(y_{E}-L_{y}\right)\right]}{\sqrt{\left(x_{E}-L_{x}\right)^{2}+\left(y_{E}-L_{y}\right)^{2}+z_{E}^{2}}}\right\}, \\
& \delta=3\left\{\frac{(1-\mu)\left[\left(x_{E}+\mu\right) z_{E}\right]}{\sqrt{\left(x_{E}+\mu\right)^{2}+y_{E}^{2}+z_{E}^{2}}}+\frac{\mu\left[\left(x_{E}+\mu-1\right) z_{E}\right]}{\sqrt{\left(x_{E}+\mu-1\right)^{2}+y_{E}^{2}+z_{E}^{2}}}+\frac{\epsilon\left[\left(x_{E}-L_{x}\right) z_{E}\right]}{{\sqrt{\left(x_{E}-L_{x}\right)^{2}+\left(y_{E}-L_{y}\right)^{2}+z_{E}^{2}}}^{5}}\right\}, \\
& \phi=3\left\{\frac{(1-\mu)\left[y_{E} z_{E}\right]}{\sqrt{\left(x_{E}+\mu\right)^{2}+y_{E}^{2}+z_{E}^{2}}} 5+\frac{\mu\left[y_{E} z_{E}\right]}{\sqrt{\left(x_{E}+\mu-1\right)^{2}+y_{E}^{2}+z_{E}^{2}}}+\frac{\epsilon\left[\left(y_{E}-L_{y}\right) z_{E}\right]}{{\sqrt{\left(x_{E}-L_{x}\right)^{2}+\left(y_{E}-L_{y}\right)^{2}+z_{E}^{2}}}^{5}}\right\} .
\end{aligned}
$$

Calling $\mathcal{A}$ the matrix corresponding to system (4), namely:

$$
\mathcal{A}=\left(\begin{array}{llllll}
0 & 0 & 0 & 1 & 0 & 0 \\
0 & 0 & 0 & 0 & 1 & 0 \\
0 & 0 & 0 & 0 & 0 & 1 \\
\alpha & \chi & \delta & 0 & 2 & 0 \\
\chi & \beta & \phi & -2 & 0 & 0 \\
\delta & \phi & \gamma & 0 & 0 & 0
\end{array}\right)
$$

The characteristic polynomial of $\mathcal{A}$ is

$$
\begin{aligned}
& \Psi^{6}+(4-\alpha-\beta-\gamma) \Psi^{4}+\left(\alpha \beta+\alpha \gamma+\beta \gamma-\chi^{2}-\delta^{2}-\phi^{2}-4 \gamma\right) \Psi^{2}+\left(\alpha \phi^{2}+\beta \delta^{2}\right. \\
& \left.\quad+\gamma \chi^{2}-\alpha \beta \gamma-2 \chi \delta \phi\right)=0
\end{aligned}
$$

As (7) is a biquadratic equation it is useful to set $\Gamma=\Psi^{2}$, and, observing that $(4-$ $\alpha-\beta-\gamma)=2$ in (5), yields the monic polynomial of the third degree:

$$
\begin{aligned}
& \Gamma^{3}+2 \Gamma^{2}+\left(\alpha \beta+\alpha \gamma+\beta \gamma-\chi^{2}-\delta^{2}-\phi^{2}-4 \gamma\right) \Gamma+\left(\alpha \phi^{2}+\beta \delta^{2}+\gamma \chi^{2}-\alpha \beta \gamma\right. \\
& \quad-2 \chi \delta \phi)=0 .
\end{aligned}
$$

Following the usual procedure to solve third degree polynomials, see for example, Artin (1991), we call

$$
\begin{aligned}
& p=-\frac{4}{3}+\left(\alpha \beta+\alpha \gamma+\beta \gamma-\chi^{2}-\delta^{2}-\phi^{2}-4 \gamma\right), \\
& q=\frac{16}{27}-\frac{2\left(\alpha \beta+\alpha \gamma+\beta \gamma-\chi^{2}-\delta^{2}-\phi^{2}-4 \gamma\right)}{3}+\left(\alpha \phi^{2}+\beta \delta^{2}+\gamma \chi^{2}-\alpha \beta \gamma-2 \chi \delta \phi\right), \\
& \Delta=\frac{q^{2}}{4}+\frac{p^{3}}{27} .
\end{aligned}
$$

Then it is well known that the three solutions $\Gamma_{1,2,3}$ of (8) are the only three solutions among those nine

$$
\Gamma_{1,2,3}=\sqrt[3]{-\frac{q}{3}+\sqrt{\Delta}}+\sqrt[3]{-\frac{q}{3}-\sqrt{\Delta}}-\frac{2}{3}
$$

such as

$$
\left(\sqrt[3]{-\frac{q}{2}+\sqrt{\Delta}}\right) \cdot\left(\sqrt[3]{-\frac{q}{2}-\sqrt{\Delta}}\right)=-\frac{p}{3} \in \mathbb{R}
$$


This means that the six eigenvalues of system (4) will be $\Psi_{k}, \quad k=1, \ldots, 6$ defined as:

$$
\Psi_{k}= \pm \sqrt{\Gamma_{j}} \quad k=1, \ldots, 6 ; j=1,2,3 .
$$

By the stability result for unequal masses of Baltagiannis and Papadakis (2011), there exist a lower limit for the mass ratio $\frac{m_{1}}{m_{1}+m_{2}}(=1-\mu)$ such as, for all the values bigger than that, the points $M_{6}$ and $M_{7}$ are always stable, which happens, for example, when fixing the three massive bodies in our model to be the Sun and any other two objects of the Solar System. Moreover, as such ratio decreases with the increase in mass of the second primary, we fix $\frac{m_{1}}{m_{1}+m_{2}}$ to be the minimal obtainable for the solar system, or, equivalently, we choose $P_{2}$ to be Jupiter (i.e. $\mu=0.000953592,1-\mu=0.999046$ ), configuration that, in particular, satisfies the stability condition $\frac{(1-\mu)}{\mu}>25+18 \sqrt{2}$ that can also be rearranged as: $\mu<\frac{(13-9 \sqrt{2})}{14}$.

From now on, we will consider the main primaries of our system to be the Sun and Jupiter; therefore, fixing a specific mass $\epsilon \in(0, \mu)$ for the hypothetical asteroid, and evaluating the eigenvalues corresponding to both the equilibrium points $M_{4}$ and $M_{5}$, the $\Delta$ will be negative, while $\sqrt{-3 p}$ will be greater than one, which, as will be shown later on, means that at least one of the three eigenvalues will have Real part non-zero, i.e. the equilibrium points are linearly unstable and therefore nonlinearly unstable; on the other hand for both the equilibrium points $M_{6}$ and $M_{7}$, the $\Delta$ will be negative, and $\sqrt{-3 p}$ will be smaller than one, which, implies that all the eigenvalues will be purely imaginary, i.e. the equilibrium points are linearly stable.

\section{The low-thrust autonomous coplanar CRFBP}

The dynamics of a low-thrust spacecraft in the autonomous coplanar CRFBP is now investigated. Using SEP propulsion our spacecraft can create artificial equilibrium points in the spatial vicinity of the asteroid, suitable for observation missions. In addition, a subset of these novel equilibrium points are proved to be stable, such that the motion will remain bounded in a small region about them, with relatively low fuel requirements and without the need for a state feedback control.

Given a maximum thrust capability $\mathcal{F}_{\max }$, expressed in $N$, and an approximate weight for the spacecraft $W_{s}$, evaluated in $k g$, the maximal acceleration in the nondimensional units is given by:

$$
\begin{aligned}
a_{\max }= & \frac{\mathcal{F}_{\max }}{W_{s}} \frac{N}{k g}=\frac{\mathcal{F}_{\max }}{W_{s}} \cdot \frac{m}{s^{2}} \\
& =\frac{\mathcal{F}_{\max }}{W_{s}} \cdot \frac{K g}{m^{2}} \frac{m^{3}}{K g \cdot s^{2}} \\
& =\frac{\mathcal{F}_{\max }}{W_{s}} \frac{d_{P_{1} / P_{2}}^{2}}{\left(m_{1}+m_{2}\right)} \frac{1}{\mathcal{G}},
\end{aligned}
$$

where $d_{P_{1} / P_{2}}$ means the distance in meters between the two major Primaries.

The acceleration will be indicated with $a \hat{\mathbf{n}}=a_{x} \overline{\mathbf{x}}+a_{y} \overline{\mathbf{y}}+a_{z} \overline{\mathbf{z}}$, where $a_{x}, a_{y}$, and $a_{z}$ are the components of the acceleration in the $x, y$ and $z$ directions, $a=\sqrt{a_{x}^{2}+a_{y}^{2}+a_{z}^{2}}$ is the magnitude and $\hat{\mathbf{n}}$ is the direction of the acceleration itself.

Considering the actual thrust capability range between $0.001 N$ to $0.3 N$, as stated by the results of the "UK ion propulsion programme" (Fearn and Crookham, 2005), the maximal reachable thrust $\mathcal{F}_{\max }$ is initially set to be $0.3 N$. Moreover, in order to estimate the range of possible acceleration on the spacecraft it's mass is set to be $1000 \mathrm{~kg}$. For the Sun-Jupiter-Asteroid-Spacecraft system, the non-dimensional value of 
the maximum acceleration $a_{\max }$ (corresponding to the maximal thrust $\mathcal{F}_{\max }=0.3 N$ ) will therefore be 1.36765 . Moreover the acceleration has to be constant in the direction of the perturbation, namely

$$
\frac{\partial}{\partial \mathbf{x}}(a \hat{\mathbf{n}})=\frac{\partial}{\partial \mathbf{y}}(a \hat{\mathbf{n}})=\frac{\partial}{\partial \mathbf{z}}(a \hat{\mathbf{n}})=0 .
$$

Adding low-thrust to system (1) it becomes:

$$
\left\{\begin{array}{l}
\ddot{x}=2 \dot{y}-\frac{\partial \Omega}{\partial x}+a_{x} \\
\ddot{y}=-2 \dot{x}-\frac{\partial \Omega}{\partial y}+a_{y} \\
\ddot{z}=-\frac{\partial \Omega}{\partial z}+a_{z}
\end{array}\right.
$$

with $\Omega, a, r_{1}, r_{2}$ and $r_{3}$ as in (1).

Again, to find the equilibrium points, the velocities $\dot{x}, \dot{y}, \dot{z}$ and the accelerations $\ddot{x}, \ddot{y}, \ddot{z}$ are set to be zero in (15), obtaining:

$$
\left\{\begin{array} { l } 
{ a _ { x } = \frac { \partial \Omega } { \partial x } } \\
{ a _ { y } = \frac { \partial \Omega } { \partial y } } \\
{ a _ { z } = \frac { \partial \Omega } { \partial z } }
\end{array} \Leftrightarrow \left\{\begin{array}{l}
a=|\nabla \Omega| \\
\hat{\mathbf{n}}=-\frac{\nabla \Omega}{|\nabla \Omega|}
\end{array}\right.\right.
$$

In which, the second system states that, in order to get a new equilibrium point, the acceleration on the spacecraft due to the thrusters has to be equal in magnitude (first equation) but opposite in direction (second equation) to the acceleration on the spacecraft due to the gravitational field at that point; the sign of the three components $a_{x}, a_{y}$ and $a_{z}$ will therefore be determined (and, in particular, opposite) by the respective components of the gravitational field evaluated in the point.

\section{Stability analysis of the linearized system}

Notice that, with a constant thrust, system (15), once linearized, is equal to the linear system in (4) and therefore the linear stability of the equilibrium points resulting from system (16) will be given by the analysis of the eigenvalues in (12). By the Lyapunov Stability theorem, see for example Arnold et al. (2006), in order to obtain a linearly bounded motion, the eigenvalues must have Real part less than or equal to zero. In our case, we cannot accept a non zero Real part, as it would imply that either $\operatorname{Re}\left(\Psi_{2 k-1}\right)>$ 0 or $\operatorname{Re}\left(\Psi_{2 k}\right)=\operatorname{Re}\left(-\Psi_{2 k-1}\right)=-\operatorname{Re}\left(\Psi_{2 k-1}\right)>0$ for $k=1$ and/or 2 and $/$ or 3 , and this would lead to a saddle $\times$ saddle $\times$ saddle, a saddle $\times$ saddle $\times$ center or a saddle $\times$ center $\times$ center unstable equilibrium point.

Therefore, in this case, the only acceptable way to get a linearly bounded motion is with $\operatorname{Re}\left(\Psi_{k}\right)=0, \forall k=1, \ldots, 6$.

Lemma 1 If $\Delta<0$ then the solutions $\Gamma_{j}, \quad j=1,2,3$ of (8) are in $\mathbb{R}$.

Proof:

Let be $\Delta<0$.

From (10) and (11), and considering the fact that $\Delta<0$, the solutions $\Gamma_{j}, \quad j=1,2,3$ of (8) can be rearranged to yield the three solutions among these nine

$$
\Gamma_{1,2,3}=\sqrt[3]{-\frac{q}{3}+i \sqrt{-\Delta}}+\sqrt[3]{-\frac{q}{3}-i \sqrt{-\Delta}}-\frac{2}{3}
$$


that satisfy the condition:

$$
\left(\sqrt[3]{-\frac{q}{2}+i \sqrt{-\Delta}}\right) \cdot\left(\sqrt[3]{-\frac{q}{2}-i \sqrt{-\Delta}}\right)=-\frac{p}{3} \in \mathbb{R}
$$

Notice that, from system (9),

$$
\Delta<0 \Rightarrow p<0
$$

and that the two numbers

$$
\begin{aligned}
& -\frac{q}{2}+i \sqrt{-\Delta} \\
& -\frac{q}{2}-i \sqrt{-\Delta}
\end{aligned}
$$

are complex conjugates (same Real part, opposite Imaginary part) such that:

$$
\left|-\frac{q}{2}+i \sqrt{-\Delta}\right|=\left|-\frac{q}{2}-i \sqrt{-\Delta}\right|=\sqrt{\left(-\frac{q}{2}\right)^{2}-(\sqrt{-\Delta})^{2}}=\sqrt{-\frac{p}{3}} \in \mathbb{R}
$$

Calling

$$
\theta=\left\{\begin{array}{l}
\arctan \left(-\frac{2 \sqrt{-\Delta}}{q}\right) \quad \text { if } \quad q<0 \\
\arctan \left(-\frac{2 \sqrt{-\Delta}}{q}\right)-\pi \text { if } q>0
\end{array}\right.
$$

the two numbers can be rewritten as:

$$
\begin{aligned}
& \sqrt{-\frac{p^{3}}{27}}\left(e^{i \theta}\right) \\
& \sqrt{-\frac{p^{3}}{27}}\left(e^{-i \theta}\right)
\end{aligned}
$$

Thus, extracting the cubic root, gives:

$$
\begin{aligned}
& \sqrt{-\frac{p}{3}}\left(e^{i\left(\frac{\theta}{3}+\frac{2 k \pi}{3}\right)}\right) \\
& \sqrt{-\frac{p}{3}}\left(e^{i\left(-\frac{\theta}{3}+\frac{2 h \pi}{3}\right)}\right)
\end{aligned}
$$

with $h, k=0,1,2$.

For condition (18), we can only accept the couples $(h=0 ; k=0),(h=2 ; k=1)$ and $(h=1 ; k=2)$. This implies that our three acceptable solutions of (8) can be summarized in a compact form as:

$$
\begin{aligned}
\Gamma_{j}= & \sqrt{-\frac{p}{3}}\left(e^{i\left(\frac{\theta}{3}+\frac{2(j-1) \pi}{3}\right)}+e^{i\left(-\frac{\theta}{3}+\frac{4(j-1) \pi}{3}\right)}\right)-\frac{2}{3} \\
= & \sqrt{-\frac{p}{3}}\left(\cos \left(\frac{\theta}{3}+\frac{2(j-1) \pi}{3}\right)+i \sin \left(\frac{\theta}{3}+\frac{2(j-1) \pi}{3}\right)\right) \\
& +\sqrt{-\frac{p}{3}}\left(\cos \left(\frac{\theta}{3}+\frac{2(j-1) \pi}{3}\right)-i \sin \left(\frac{\theta}{3}+\frac{2(j-1) \pi}{3}\right)\right)-\frac{2}{3} \\
= & 2 \sqrt{-\frac{p}{3}} \cos \left(\frac{\theta}{3}+\frac{2(j-1) \pi}{3}\right)-\frac{2}{3},
\end{aligned}
$$

with $j=1,2,3$.

Which demonstrates that $\Delta<0 \Rightarrow \Gamma_{j} \in \mathbb{R}, \quad \forall j=1,2,3 \square$.

Lemma 2 If $\sqrt{-3 p}<1$ then $\Gamma_{j}<0, \quad \forall j=1,2,3$. 
Proof:

By (25) for each $j=1,2,3$ we can rewrite $\Gamma_{j}$ as

$$
\Gamma_{j}=2 \sqrt{-\frac{p}{3}} \cos \left(\frac{\theta}{3}+\frac{2(j-1) \pi}{3}\right)-\frac{2}{3} .
$$

Then, for hypothesis:

$$
2 \sqrt{-\frac{p}{3}} \cos \left(\frac{\theta}{3}+\frac{2(i-1) \pi}{3}\right)-\frac{2}{3} \leq 2 \sqrt{-\frac{p}{3}}-\frac{2}{3}<\frac{2}{3}-\frac{2}{3}=0
$$

Lemma 3 If

$$
\left\{\begin{array}{l}
\Delta<0 \\
\sqrt{-3 p}<1
\end{array}\right.
$$

then the six eigenvalues of system (4) are purely Imaginary.

Proof:

The proof of the Lemma is straight forward since the eigenvalues of (4) are $\Psi_{k}=$ $\pm \sqrt{\Gamma_{j}}, \quad k=1, \ldots, 6 ; \quad j=1,2,3$. Considering the hypothesis and applying Lemma 1, indeed, yields that $\Gamma \in \mathbb{R}, \forall j=1,2,3$. Then, for Lemma $2, \Gamma_{j}<0, \forall j=1,2,3$, which are the two conditions that lead to the thesis of Lemma $3 \square$.

Therefore, the six eigenvalues can be rearranged in the form:

$$
\Psi_{k}= \pm i \sqrt{-\Gamma_{j}}, \quad k=1, \ldots, 6 ; \quad j=1,2,3 .
$$

Figure 5 shows the spatial view of the three possible topologies of the zone that satisfies the conditions in (28), obtained for a realistic range for the mass of the hypothetical asteroid (i.e. from zero to the total mass of the Jupiter Trojans $\sim 6 \times 10^{20} \mathrm{~kg}$ ).

In particular part a) of the Figure represents the topology of the "four leaf clover" (Ceccaroni and Biggs, 2010) for $m_{3} \in\left[0 ; 1.648 \times 10^{17}\left[\left(\Rightarrow \epsilon \in\left[0 ; 8.27632 \times 10^{-14}[)\right.\right.\right.\right.$, part b) represents it for $m_{3}=1.648 \times 10^{17}\left(\Rightarrow \epsilon=8.27632 \times 10^{-14}\right)$, and part c) for $\left.\left.\left.\left.m_{3} \in\right] 1.648 \times 10^{17} ; 6 \times 10^{20}\right](\Rightarrow \epsilon \in] 8.27632 \times 10^{-14} ; 3.01356 \times 10^{-10}\right]\right)$.

In this figure, the zone that satisfies the conditions in (28), is the linearly "stable zone", intersection of the zone outside the dashed, dark, meshed surfaces and that outside the continuous, light, meshed surfaces which represents the solution of the first and the second equations of system (28) respectively. 


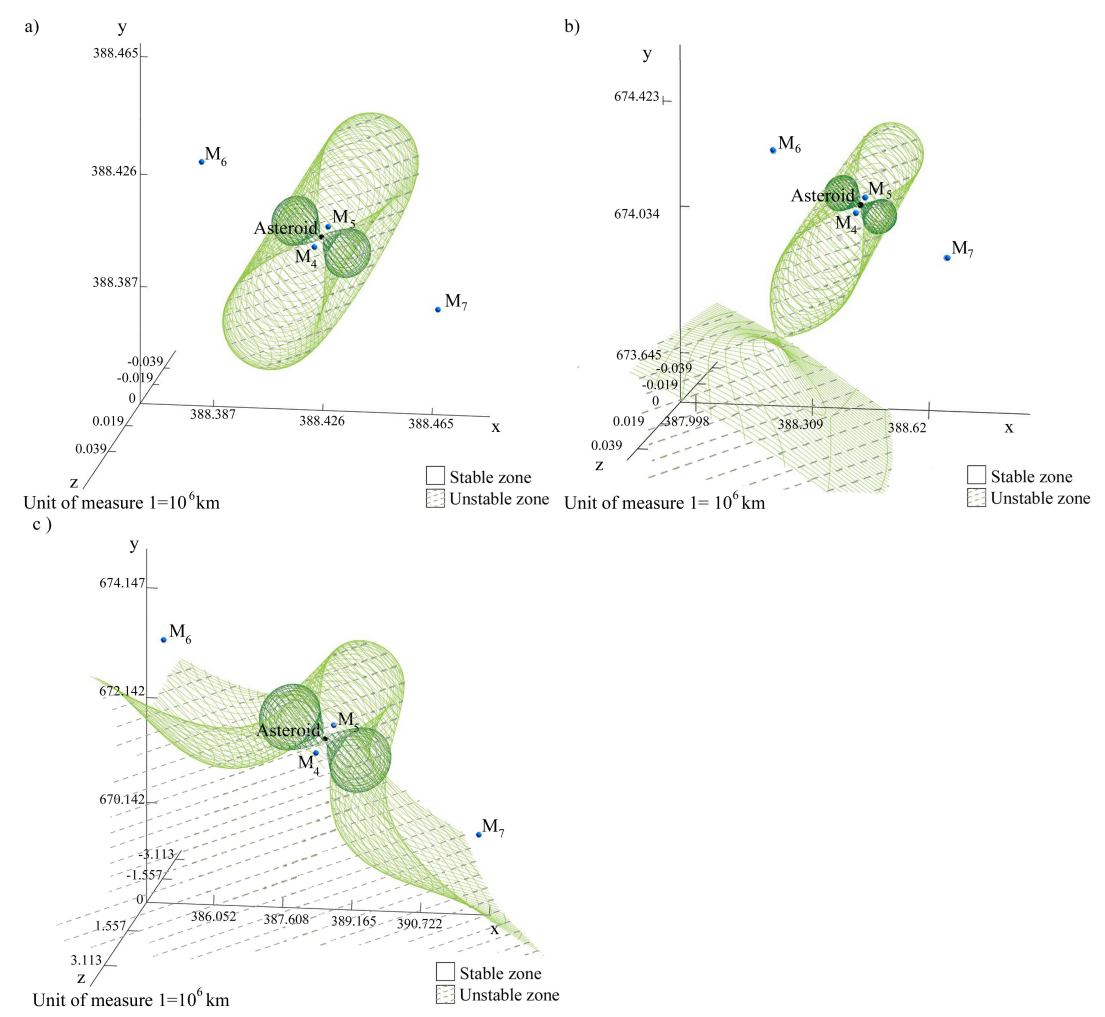

Fig. 5 The possible topologies obtainable varying the mass of the Asteroid; $a) m_{3} \in[0 ; 1.648 \times$ $10^{17}\left[\left(\Rightarrow \epsilon \in\left[0 ; 8.27632 \times 10^{-14}[) . b\right) m_{3}=1.648 \times 10^{17}\left(\Rightarrow \epsilon=8.27632 \times 10^{-14}\right) . c\right)\right.$ $\left.\left.\left.\left.m_{3} \in\right] 1.648 \times 10^{17} ; 6 \times 10^{20}\right](\Rightarrow \epsilon \in] 8.27632 \times 10^{-14} ; 3.01356 \times 10^{-10}\right]\right)$. 
For displaying purpose only, from now on, the mass of the Asteroid will be set to be equal to the major of the actual Trojan Asteroids, namely 624-Hektor, therefore fixing $m_{3}=1.4 \times 10^{19} \mathrm{~kg}$ (which implies $\epsilon=7.03165 \times 10^{-12}$ ). Qualitatively the results for this value will be the same as for each value in the range considered, from zero to the total mass of the Jupiter Trojans.

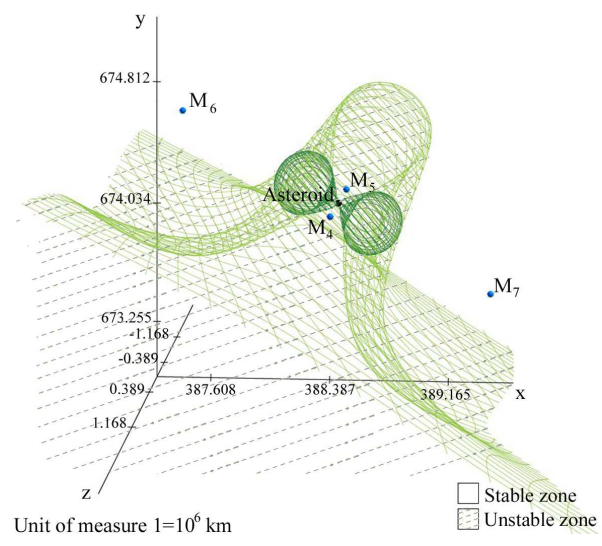

Fig. 6 Linearly stable/unstable zones; the three dimensional "four leaf clover" boundary between the linearly stable and the unstable zones near the asteroid

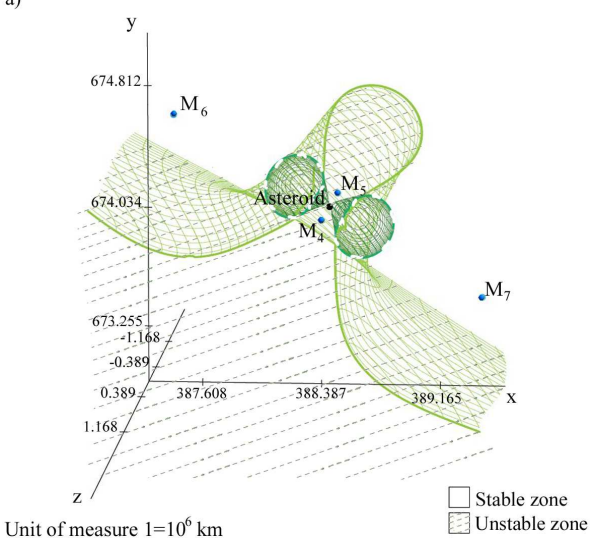

b)

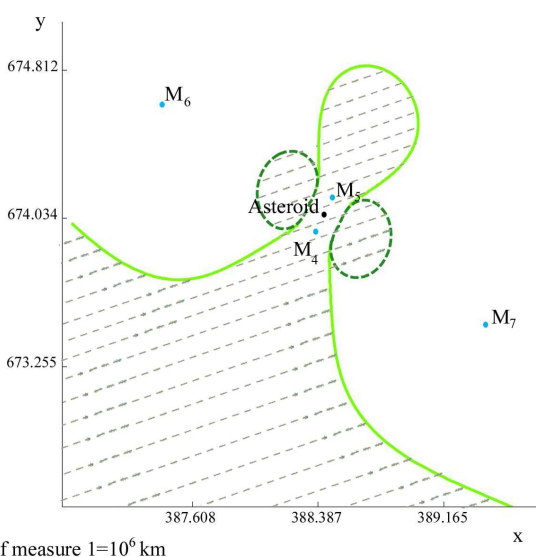

Fig. 7 Linearly stable/unstable zones; a) $z=0$ section of the spatial view; b) Intersection with the plane $z=0$

Figure 6 represents the spatial view of the linearly stable zone of the Sun-JupiterAsteroid-Spacecraft system, while, once again, Figure 7 represents the horizontal $x / y$ section, part a), and its intersection with the $x / y$ plane, part b). 
Of course, there exist an upper, external limit of the linearly stable zone as represented in Figure 8.

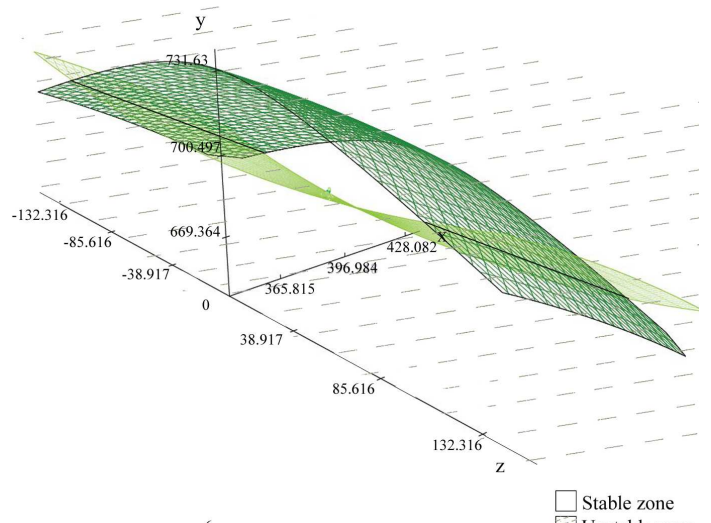

Units of measure $1=10^{6} \mathrm{~km}$

\begin{tabular}{l}
$\square$ Stable zone \\
\hline U Unstable zone
\end{tabular}

Fig. 8 The outside boundary; The linearly stable zone is bounded.

a)

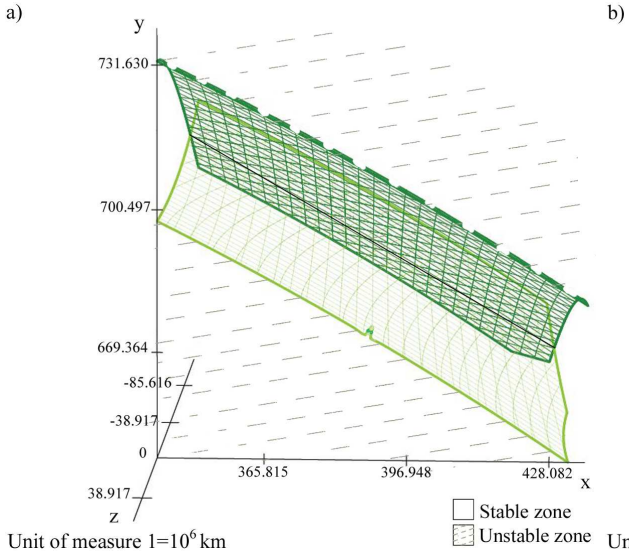

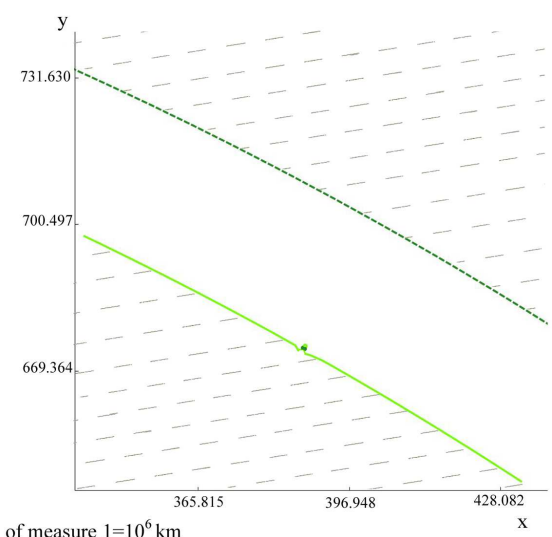

Fig. 9 The outside boundary; a) $z=0$ section of the spatial view b) Intersection with the plane $z=0$

In particular the first inequality of system (28) is verified by the points between the dashed, dark, meshed surfaces, while the second by those between the continuous, light, meshed surfaces. Thus, within the bounded area defined by the intersection of these two zones the linearized motion is stable. Once again, these zones are represented in the three dimensions, Figure 8, then sectioned with the $z=0$ plane, Figure 9 a), and finally intersected with this plane, Figure $9 \mathrm{~b}$ ). 
With reference to Figure 5 we should note that the external limit is separated from the four leaf clover for small values of the mass of the asteroid, then approaches it as the mass of the Asteroid grows to $1.648 \times 10^{17} \mathrm{~kg}$, where two surfaces becomes tangent, and finally, as the mass overcomes such value, the two surfaces merge together and the lower part of the four leaf clover opens.

The maximum possible thrust required to overcome the lower outside boundary is about $7 \times 10^{-4} N$ while for the upper one the thrust required is about $2.5 \times 10^{-2} N$. However, for the system considered, the maximum thrust actually needed for this simplified model will be lower than $1.5 \times 10^{-4} \mathrm{~N}$.

For the purpose of exposition, eight linearly stable artificial equilibrium points $A, B, C$, $D, E, F, G, H$ are chosen, which have to fulfill three main requirements: to be generated by a thrust lower than $1.5 \times 10^{-4} N$, to be at a distance from the unstable zone equal or higher than $3500 \mathrm{~km}$ (far enough from the unstable zone to account for the likelihood of injection/position errors of the spacecraft), but remaining as close as possible to the asteroid. Moreover we take the first four lying on the $x / y$ plane, while the other on the $x^{\prime} / z$ plane where $x^{\prime}$ is the tilted horizontal axis $y=-(x-$ $\left.L_{x}\right) \frac{M_{5 x}-M_{4 x}}{M_{5 y}-M_{4 y}}+L_{y}$, (i.e. the line passing through the asteroid and perpendicular to the segment connecting $M_{4}$ and $M_{5}$ ). The eight points (whose coordinates are listed in the electronic supplementary material A (online only)) result to be at a distance smaller than $300000 \mathrm{~km}$ from the asteroid (note that the distance of the stable equilibrium points from the Asteroid is about $1.16 \times 10^{6} \mathrm{~km}$, more that two thirds bigger than the distance of the asteroid from the artificial equilibrium points chosen).

In this system, in non-dimensional units, the amount of thrust required to create the two artificial points in $A=\left(A_{x} ; A_{y} ; A_{z}\right)$ and in $G=\left(G_{x} ; G_{y} ; G_{z}\right)$ are evaluated; the behavior of the dynamics close to these two artificial equilibrium points, as well as their required thrust, are representative of the four points on the $z=0$ plane, and for the four on the $x^{\prime} / z$ plane respectively, as can be seen in Figures 11 and 12, and, therefore, will be the only two investigated in detail.

To this end the computation of the gravitational field in both $A$ and $G$ is needed:

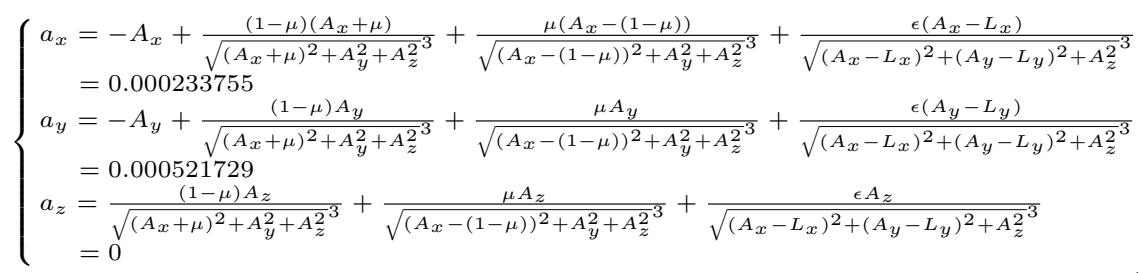

which yields to $a=\sqrt{a_{x}^{2}+a_{y}^{2}+a_{z}^{2}}=0.000571701$ corresponding approximately to a force of $0.000125406 N$; the same evaluation for the point $G$ leads to $\left(a_{x}, a_{y}, a_{z}\right)=$ $(0.0000240703,-0.00001415,0.00034275)$ which is $a=\sqrt{a_{x}^{2}+a_{y}^{2}+a_{z}^{2}}=0.000343885$ corresponding approximately to a force of $0.000075433 \mathrm{~N}$.

These thrusts are represented respectively by the two "tube shaped", meshed surfaces in Figures 10, 11 and 12; the first thrust is approximately the same required to create the other three equilibrium points lying on the $x / y$ plane $(B, C$, and $D)$, while the other is approximately the same required to create artificial equilibrium points set on the $x^{\prime} / z$ plane $(E, F$, and $H)$. Figure $11 \mathrm{a}$ ) and b) represents the horizontal $x / y$ section and the intersection with the $x / y$ plane of Figure 10.

As the four leaf clover is tilted with respect to the system of reference, it is also useful, 
in terms of visual presentation, to section and intersect it with the vertical plane $x^{\prime} / z$ as shown in Figure 12 a) and b) respectively.

Note that, the eight points in Figures 10, 11 and 12 lie $3500 \mathrm{~km}$ outside the linearly unstable zone, although it is not easy to visualize.

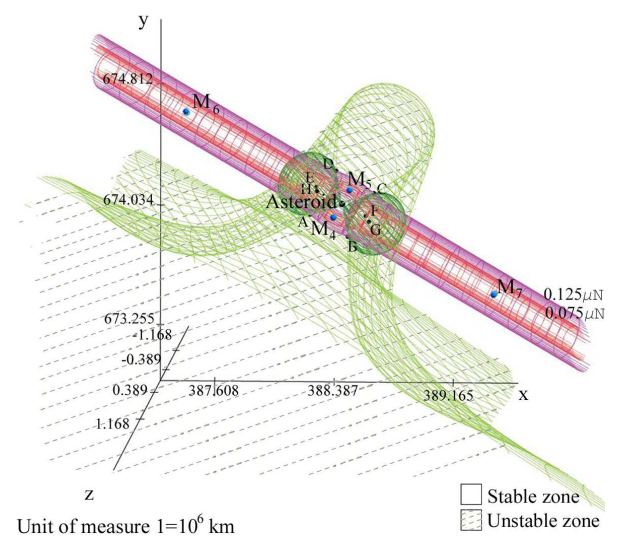

Fig. 10 Adding the low-thrust; eight artificial equilibrium points are created in the linearly stable zone using a thrust lower than $1.5 \times 10^{-4}$

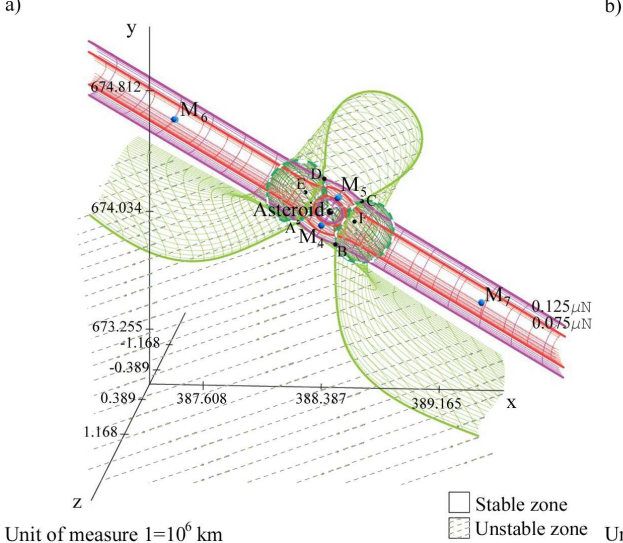

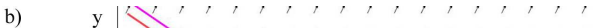

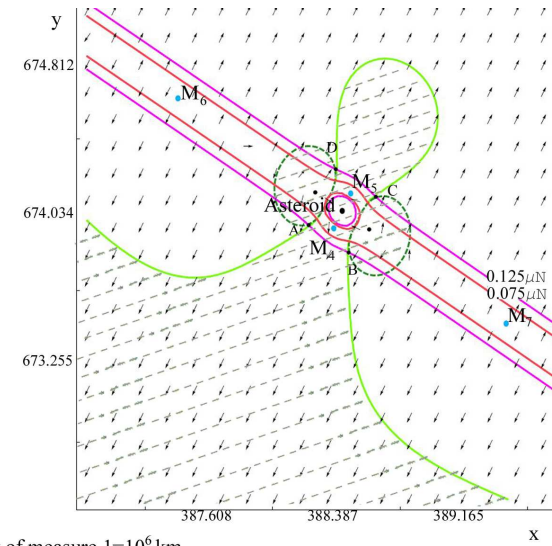

Unstable zone Unit of measure $1=10^{6} \mathrm{~km}$

Fig. 11 Adding the low-thrust; a) $z=0$ section of the spatial view; b) Intersection with the plane $z=0$ and projection on this plane of the direction of the thrust required

Moreover, parts b) of Figures 11 and 12 show the projections, on the respective planes, of the directions of the thrust required to counterbalance the gravitational field creating artificial equilibrium points. 

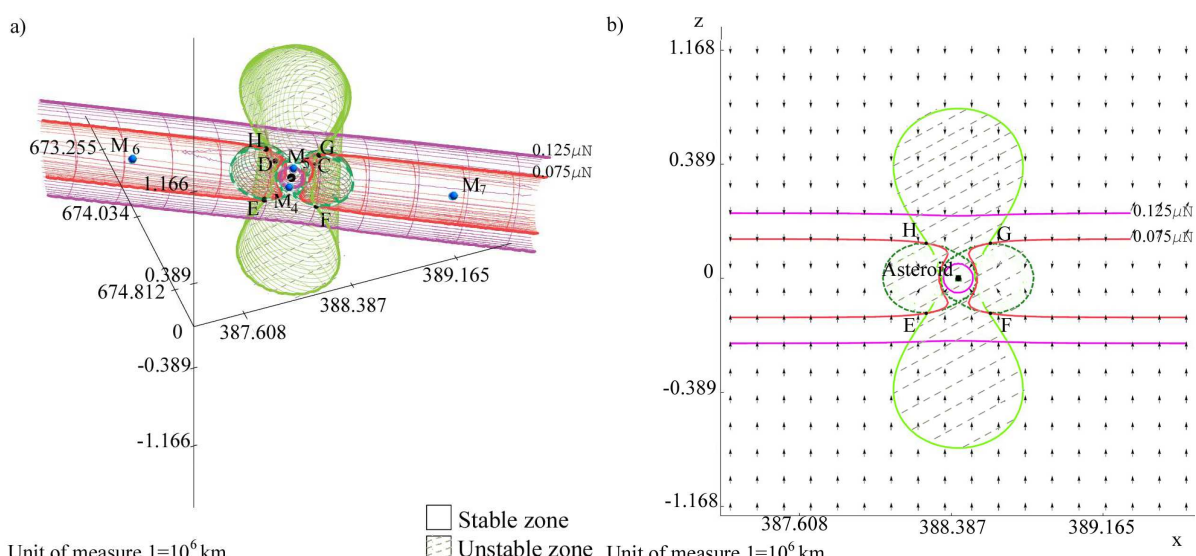

Unit of measure $1=10^{6} \mathrm{~km}$

Fig. 12 Adding the low-thrust; a) $x^{\prime} / z$ section of the spatial view; b) Intersection with the plane $x^{\prime} / z$ and projection on this plane direction of the direction of the thrust required

\section{Integrating the linearized motion}

Theorem 1 For a linear system of the form:

$$
\left\{\begin{array}{l}
\dot{\mathrm{x}}=\mathcal{A} \mathrm{x} \\
\mathrm{x}\left(t_{0}\right)=\mathrm{x}_{0}
\end{array}\right.
$$

where $\mathrm{x} \in \mathbb{R}^{6}$ and $\mathcal{A} \in \mathcal{L}\left(\mathbb{R}^{6}\right):=\left\{\right.$ linear operators $\mathcal{A}: \mathbb{R}^{6} \rightarrow \mathbb{R}^{6}$, with real coefficients $\}$, if $\mathcal{A}$ has three couples of eigenvalues complex conjugated $\lambda, \lambda^{*} ; \nu, \nu^{*} ; \varphi, \varphi^{*} \in \mathbb{C}$, where the complex conjugated is indicated by the ${ }^{*}$, such that:

$$
\begin{aligned}
& \lambda=\lambda_{R}+i \lambda_{I}, \\
& \nu=\nu_{R}+i \nu_{I}, \\
& \varphi=\varphi_{R}+i \varphi_{I},
\end{aligned}
$$

where $\lambda_{R}=\operatorname{Re}(\lambda)$ and $\lambda_{I}=\operatorname{Im}(\lambda)$ and $\lambda_{I}, \nu_{I}, \varphi_{I} \neq 0$; then there exist a base of vectors $\left\{\mathbf{u}_{1}, \mathbf{w}_{1}, \mathbf{u}_{3}, \mathbf{w}_{3}, \mathbf{u}_{5}, \mathbf{w}_{5}\right\}$, with $\mathbf{u}_{2 j-1}, \mathbf{w}_{2 j-1} \in \mathbb{R}^{6} \forall j=1,2,3$, and where $\mathbf{f}_{2 j-1}=\mathbf{u}_{2 j-1}+i \mathbf{w}_{2 j-1}, \quad j=1,2,3$ are the eigenvectors of system (31), such that, in this basis, the solution $\Xi(t)=\left[\xi_{1}(t), \xi_{2}(t), \psi_{1}(t), \psi_{2}(t), \zeta_{1}(t), \zeta_{2}(t)\right]^{T}$ of the problem is:

$$
\left\{\begin{array}{l}
\xi_{1}(t)=e^{\lambda_{R} t}\left(\cos \left(\lambda_{I} t\right) \xi_{1}^{0}+\sin \left(\lambda_{I} t\right) \xi_{2}^{0}\right) \\
\xi_{2}(t)=e^{\lambda_{R} t}\left(\cos \left(\lambda_{I} t\right) \xi_{2}^{0}-\sin \left(\lambda_{I} t\right) \xi_{1}^{0}\right) \\
\psi_{1}(t)=e^{\nu_{R} t}\left(\cos \left(\nu_{I} t\right) \psi_{1}^{0}+\sin \left(\nu_{I} t\right) \psi_{2}^{0}\right) \\
\psi_{2}(t)=e^{\nu_{R} t}\left(\cos \left(\nu_{I} t\right) \psi_{2}^{0}-\sin \left(\nu_{I} t\right) \psi_{1}^{0}\right) \\
\zeta_{1}(t)=e^{\varphi_{R} t}\left(\cos \left(\varphi_{I} t\right) \zeta_{1}^{0}+\sin \left(\varphi_{I} t\right) \zeta_{2}^{0}\right) \\
\zeta_{2}(t)=e^{\varphi_{R} t}\left(\cos \left(\varphi_{I} t\right) \zeta_{2}^{0}-\sin \left(\varphi_{I} t\right) \zeta_{1}^{0}\right)
\end{array}\right.
$$

And therefore the solution $\mathrm{x}(t)=\left[x(t), y(t), z(t), v_{x}(t), v_{y}(t), v_{z}(t)\right]^{T}$ of system (31) will be $\mathrm{x}(t)=\mathcal{M} \Xi(t)$ where $\mathcal{M}$ is the matrix which provide the expression of the original coordinates $\mathrm{x}=\left[x, y, z, v_{x}, v_{y}, v_{z}\right]^{T}$ in terms of the new one, $\boldsymbol{J}=\left[\xi_{1}, \xi_{2}, \psi_{1}, \psi_{2}, \zeta_{1}, \zeta_{2}\right]^{T}$, i.e. is the matrix of the change of coordinates. 
Proof:

The proof of this theorem is given in Appendix B.

For the theorem above it is enough to find the eigenvectors of the system, and its solutions will be automatically obtained; moreover it will also provide the matrix $\mathcal{M}$ of the change of coordinates, as will be shown later on.

The eigenvalues of the system will be the six vectors $\mathbf{f}_{j}=\mathbf{u}_{j}+i \mathbf{w}_{j} \in \mathbb{C}^{6}, j=1, \ldots, 6$ which solve

$$
\mathcal{A} \mathbf{f}_{j}=\Psi_{j} \mathbf{f}_{j}, \quad j=1, \ldots, 6 .
$$

As the six eigenvalues are three couples of complex conjugated numbers, the eigenvectors must be three couples of complex conjugated vectors, namely $\mathbf{f}_{2 j}=\mathbf{u}_{2 j}+i \mathbf{w}_{2 j}=$ $\mathbf{u}_{2 j-1}-i \mathbf{w}_{2 j-1}=\mathbf{f}_{2 j-1}^{*}, \quad \forall j=1,2,3$. This, in particular implies that it is sufficient to find three of the six eigenvectors, i.e. $\mathbf{f}_{1}, \mathbf{f}_{3}$ and $\mathbf{f}_{5}$. Moreover, in the case considered in this paper, the eigenvalues have null Real part (i.e. are purely imaginary), and can therefore be stated in a simpler form.

Therefore, to simplify notation, hereafter the eigenvectors of the system will be:

$$
\begin{aligned}
& \Psi_{1}=\lambda i, \\
& \Psi_{2}=-\lambda i, \\
& \Psi_{3}=\nu i, \\
& \Psi_{4}=-\nu i, \\
& \Psi_{5}=\varphi i, \\
& \Psi_{6}=-\varphi i .
\end{aligned}
$$

A few algebraic manipulations of (34) lead to the determination of the coefficients $u_{k, j} \quad k=1,3,5 \quad j=1, \ldots, 6$ of the three eigenvectors corresponding to the eigenvalues $\lambda i, \nu i, \varphi i$ respectively; these coefficients are listed in the supplementary electronic material B.

Than the matrix $\mathcal{M}=\left(\mathcal{M}_{j, k}\right)_{\substack{j=1, \ldots, 6 \\ k=1, \ldots, 6}}$ of the change of coordinates will be given by

$$
\mathcal{M}_{j, k}=\left\{\begin{array}{l}
u_{k, j} \text { if } k \text { odd } \\
w_{k-1, j} \text { if } k \text { even }
\end{array}\right.
$$

Applying the transformation of coordinates $\mathcal{M}^{-1}$ on $\mathrm{x}=\left[x, y, z, v_{x}, v_{y}, v_{z}\right]^{T}$, yields the new coordinates $\boldsymbol{I}=\left[\xi_{1}, \xi_{2}, \psi_{1}, \psi_{2}, \zeta_{1}, \zeta_{2}\right]^{T}$, namely:

$$
I=\mathcal{M}^{-1} \mathrm{x} \text {. }
$$

The transformation $\mathcal{M}^{-1}$ is then performed on the system (4) to find it's expression in the new coordinates:

$$
\dot{\jmath}=\mathcal{M}^{-1} \dot{\mathrm{x}}=\mathcal{M}^{-1} \mathcal{A} \mathrm{x}=\mathcal{M}^{-1} \mathcal{A} \mathcal{M} \text { J. }
$$

Such a system can be rewritten as:

$$
\dot{\beth}=\mathcal{A}^{\prime} \beth \text { with } \mathcal{A}^{\prime}=\mathcal{M}^{-1} \mathcal{A} \mathcal{M}
$$

with

$$
\mathcal{A}^{\prime}=\left(\begin{array}{llllll}
0 & \lambda & 0 & 0 & 0 & 0 \\
-\lambda & 0 & 0 & 0 & 0 & 0 \\
0 & 0 & 0 & \nu & 0 & 0 \\
0 & 0 & -\nu & 0 & 0 & 0 \\
0 & 0 & 0 & 0 & 0 & \varphi \\
0 & 0 & 0 & 0 & -\varphi & 0
\end{array}\right)
$$


whose solutions are

$$
\left\{\begin{array}{l}
\xi_{1}(t)=\cos (\lambda t) \xi_{1}^{0}+\sin (\lambda t) \xi_{2}^{0} \\
\xi_{2}(t)=\cos (\lambda t) \xi_{2}^{0}-\sin (\lambda t) \xi_{1}^{0} \\
\psi_{1}(t)=\cos (\nu t) \psi_{1}^{0}+\sin (\nu t) \psi_{2}^{0} \\
\psi_{2}(t)=\cos (\nu t) \psi_{2}^{0}-\sin (\nu t) \psi_{1}^{0} \\
\zeta_{1}(t)=\cos (\varphi t) \zeta_{1}^{0}+\sin (\varphi t) \zeta_{2}^{0} \\
\zeta_{2}(t)=\cos (\varphi t) \zeta_{2}^{0}-\sin (\varphi t) \zeta_{1}^{0}
\end{array}\right.
$$

Therefore the solution of system (15), given by $\mathrm{x}(t)=\mathcal{M} \Xi(t)$, are:

$$
\left\{\begin{aligned}
x(t) & =\mathcal{M}_{1,1} \xi_{1}(t)+\mathcal{M}_{1,2} \xi_{2}(t)+\mathcal{M}_{1,3} \psi_{1}(t)+\mathcal{M}_{1,4} \psi_{2}(t)+\mathcal{M}_{1,5} \zeta_{1}(t)+\mathcal{M}_{1,6} \zeta_{2}(t) \\
& =u_{1,1} \xi_{1}(t)+w_{1,1} \xi_{2}(t)+u_{3,1} \psi_{1}(t)+w_{3,1} \psi_{2}(t)+u_{5,1} \zeta_{1}(t)+w_{5,1} \zeta_{2}(t) \\
y(t) & =\mathcal{M}_{2,1} \xi_{1}(t)+\mathcal{M}_{2,2} \xi_{2}(t)+\mathcal{M}_{2,3} \psi_{1}(t)+\mathcal{M}_{2,4} \psi_{2}(t)+\mathcal{M}_{2,5} \zeta_{1}(t)+\mathcal{M}_{2,6} \zeta_{2}(t) \\
& =u_{1,2} \xi_{1}(t)+w_{1,2} \xi_{2}(t)+u_{3,2} \psi_{1}(t)+w_{3,2} \psi_{2}(t)+u_{5,2} \zeta_{1}(t)+w_{5,2} \zeta_{2}(t) \\
z(t) & =\mathcal{M}_{3,1} \xi_{1}(t)+\mathcal{M}_{3,2} \xi_{2}(t)+\mathcal{M}_{3,3} \psi_{1}(t)+\mathcal{M}_{3,4} \psi_{2}(t)+\mathcal{M}_{3,5} \zeta_{1}(t)+\mathcal{M}_{3,6} \zeta_{2}(t) \\
& =u_{1,3} \xi_{1}(t)+w_{1,3} \xi_{2}(t)+u_{3,3} \psi_{1}(t)+w_{3,3} \psi_{2}(t)+u_{5,3} \zeta_{1}(t)+w_{5,3} \zeta_{2}(t)
\end{aligned}\right.
$$

where the coefficients $u_{k, j} \quad k=1,3,5 \quad j=1, \ldots, 6$ are listed in the electronic supplementary material B.

Notice that, evaluating $\mathrm{x}(0)=[x(0), y(0), z(0), \dot{x}(0), \dot{y}(0), \dot{z}(0)]^{T}$, as expected, yields:

$$
\left(\begin{array}{l}
x(0) \\
y(0) \\
z(0) \\
v_{x}(0) \\
v_{y}(0) \\
v_{z}(0)
\end{array}\right)=\mathcal{M}\left(\begin{array}{l}
\xi_{1}^{0} \\
\xi_{2}^{0} \\
\psi_{1}^{0} \\
\psi_{2}^{0} \\
\zeta_{1}^{0} \\
\zeta_{2}^{0}
\end{array}\right)
$$

which is equal to (37) evaluated at $t=0$.

Recall that these resulting orbits, solutions of the linearized system, are expressed in the system of reference translated to the artificial equilibrium point $x_{e}, y_{e}, z_{e}$ (see (3)) such that they must be translated back to the barycenter of $P_{1}$ and $P_{2}$.

As the behaviors of the linear solution in (42), in the vicinity of the points $A$ and $G$, is qualitatively the same as the other six artificial equilibria, the dynamics starting sufficiently close to these two points is shown, for the Sun-Jupiter-Asteroid-Spacecraft system, where the spacecraft has the same mass of 624-Hektor, in Figures 13 and 14.

In these Figures, the $x / y, x / z$ projections of the solution of the linearized system are represented by the dark, continuous lines. As expected, after 12 Jovian years ( 150 Earth years), they are still close to the respective starting points.

The dashed projection of the $3500 \mathrm{~km}$ spherical domains around the points are also plotted in the Figures.

Notice that, if the perturbation (the position error) on the $z$ axis was null, the orbit starting close to the point $A$ would qualitatively degenerate to the two dimensional case analyzed in Ceccaroni and Biggs (2010).

\section{Integrating the full nonlinear system}

The next step to illustrate the nonlinear stability of the linearly stable artificial equilibria identified in the previous section, is to perform a numerical integration of the full nonlinear system in their vicinity. To this end, recalling system (15), the behavior of a spacecraft starting sufficiently close to the points $A$ and $G$ is investigated. 

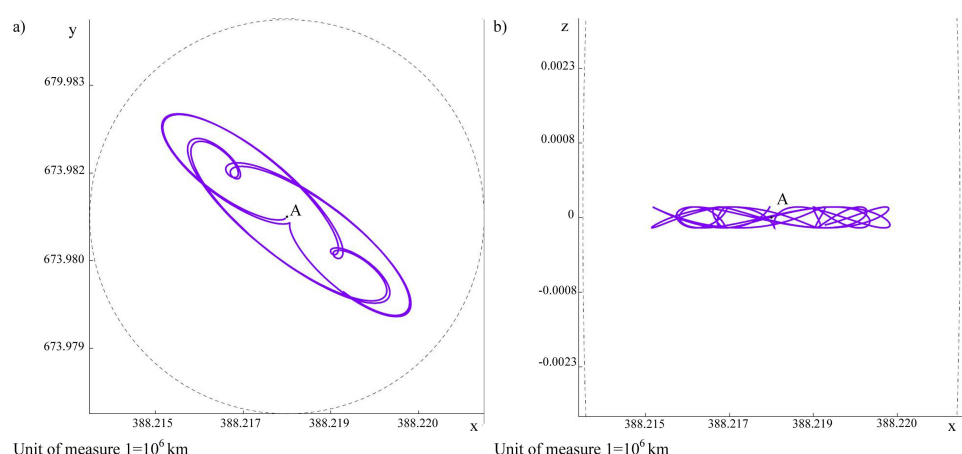

Fig. 13 The solution of the linearized system in the vicinity of the point $A$ after 12 Jovian years; a) $x / y$ projection; b) $x / z$ projection
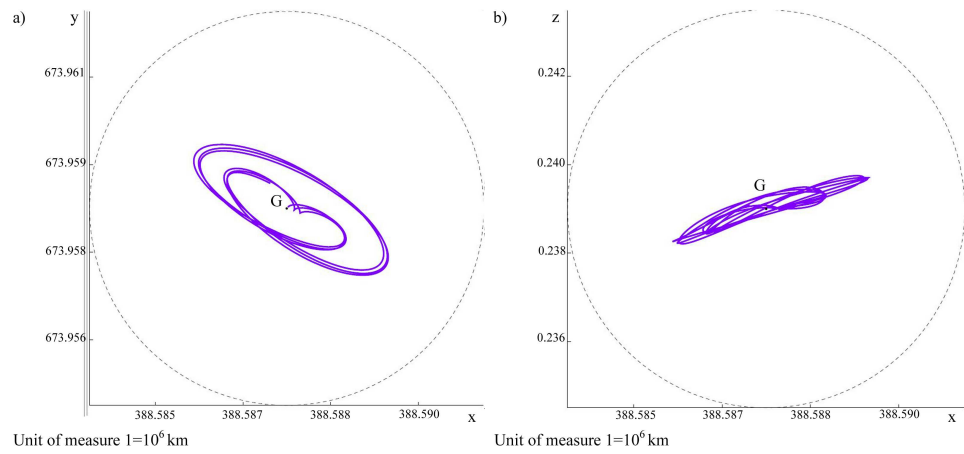

Fig. 14 The solution of the linearized system in the vicinity of the point $G$ after 12 Jovian years; a) $x / y$ projection; b) $x / z$ projection

Recalling the evaluation of the thrust needed to create artificial equilibrium points in $A$ and $G$ stated in Section 5, a numerical integration with Mathematica using a RungeKutta method is performed, starting sufficiently close to our initial points. The dark, continuous lines in Figures 15 and 16 are the $x / y$ and $x / z$ projections of the numerical solutions close to $A$ and $G$ respectively, evaluated for a null initial velocity. Once again the solutions are represented for the first 12 Jovian years and the dashed projection of the $3500 \mathrm{~km}$ spherical domains around the points appears in the Figures as well.

As a result of the application of the same method on the points $B, C, D, H, E$, and $F$, other six orbits are obtained, which, starting sufficiently close to the respective point with null initial velocity, will remain bounded around it. In particular, for approximately 150 Earth years, the motion starting close to each of the eight points, remains within the round domain, $3500 \mathrm{~km}$ radius, outside the unstable zone. Moreover the orbits obtained can be divided into four similarly-behaving couples $(\mathrm{A} / \mathrm{C}, \mathrm{B} / \mathrm{D}$, $\mathrm{H} / \mathrm{F}$ and $\mathrm{G} / \mathrm{E}$, in which, the two elements of the last two couples perfectly mirror each other), where, in particular, the motion starting close to B or D or that starting close to $\mathrm{H}$ or $\mathrm{F}$ remains bounded within an area much smaller than $3500 \mathrm{~km}$. Furthermore, looking at their behaviors it is clear that care must be taken to the choice of the initial 

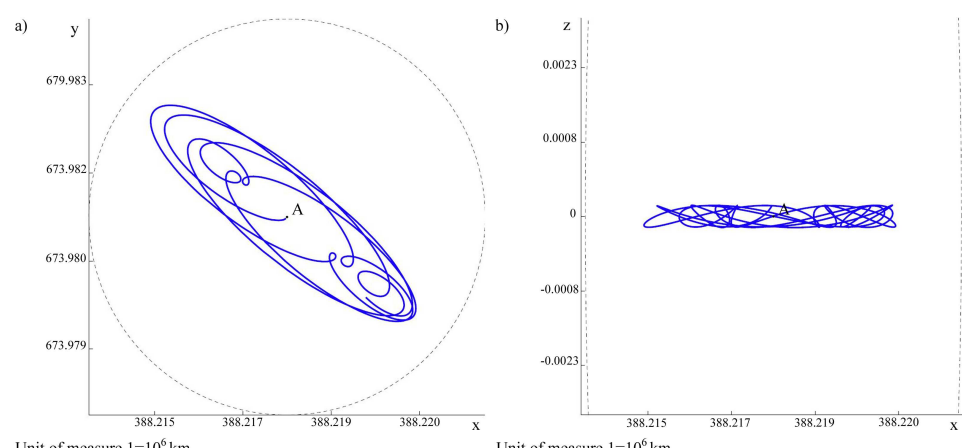

Fig. 15 The solution of the full nonlinear system in the vicinity of the point $A$ after 12 Jovian years; a) $x / y$ projection; $b$ ) $x / z$ projection
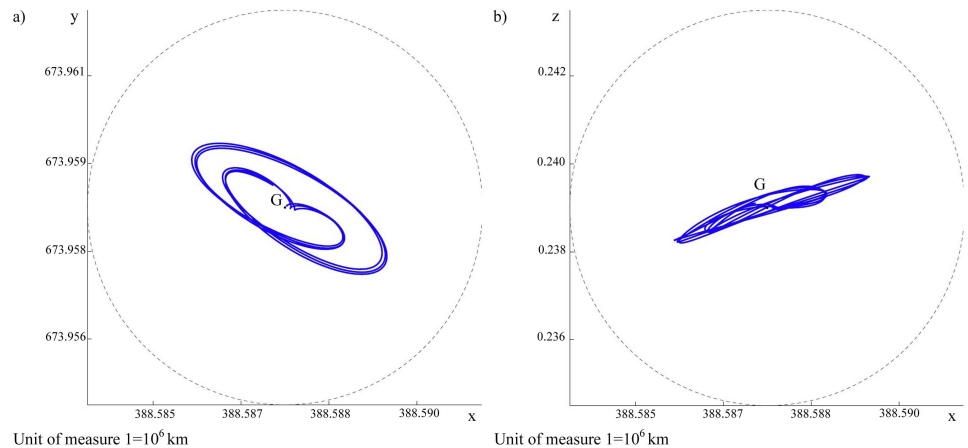

Fig. 16 The solution of the full nonlinear system in the vicinity of the point $G$ after 12 Jovian years; a) $x / y$ projection; $b$ ) $x / z$ projection

conditions as there is a trade-off between the amplitude of the oscillations about the initial point (in the $\mathrm{z}$ direction) and the fuel required, i.e. fuel can be saved at the expense of larger oscillations.

\section{The numerical simulation of the real system}

As there are no actual Jupiter Trojans in or close to $L_{4}$ a numerical simulation was required in order to compare the results found analytically in the simplified model. The actual orbits of Jupiter and 624-Hektor were derived by interpolating their daily observed position and velocity vectors (found in the JPL Small Bodies Database). In this real model the orbit of Jupiter is elliptic, and the velocity of revolution is no longer constant. Fortunately the resulting effects on the system are minimal due to the low eccentricity of Jupiter's real orbit. In addition all the tadpole orbits of the main Jupiter Trojans are inclined up to 20 degrees with respect to the plane containing Jupiter's orbit around the Sun. Thus, as the $x / y$ plane of the system of reference used is set to be the one containing the three massive planets, the change in inclination of this 
plane will greatly affect the change of velocity of the system $\dot{\omega}$ (in the theoretical model $\dot{\omega}=0$ ) therefore representing the main difference between the two models.

The eight artificial equilibria chosen in Section 6, which are bounded in the simplified model, in the real model result to be unstable, quickly diverging from 624-Hektor. The $x / y$ projections of their orbits are shown in Figure 17 for a period of around 36 years ( $\sim 3$ revolutions of Jupiter(and of Hektor) around the Sun).

The numerical integration of many other artificial equilibrium points was then under-
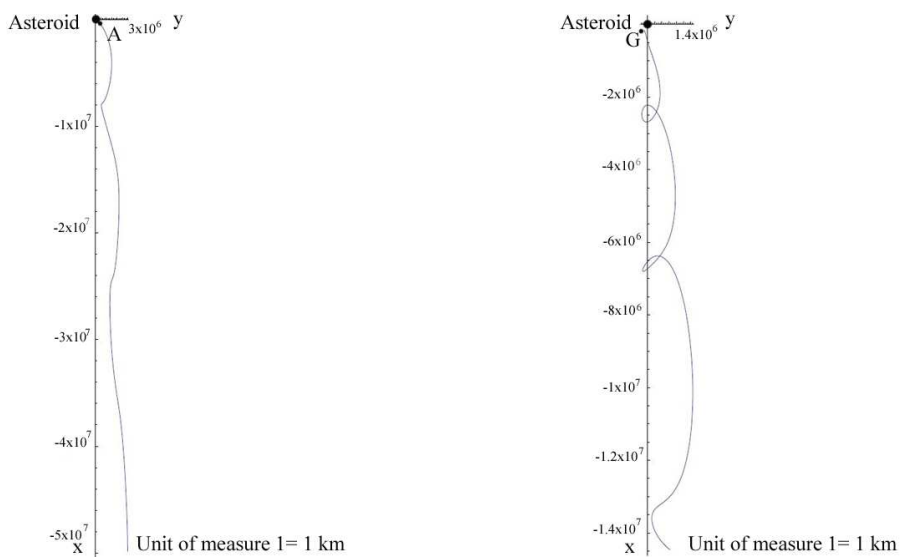

Fig. 17 The unstable zone of the model enlarges and the eight artificial equilibria above become unstable; a) The orbit generating by the point $A$ in the real system; b) The orbit generating from the point $G$ in the real system

taken. Results highlight a shift of the model towards instability, i.e. an enlargement of the unstable zone. This result is expected considering the perturbations added in the real model.

However, a zone of finite-time bounded orbits was found, in which all the orbits remain bounded within $\sim 3.5 \times 10^{6} \mathrm{~km}$ from the Asteroid for at least 36 Terrestrial years before starting to diverge. In this zone two artificial equilibria $A^{\prime}$ and $G^{\prime}$ where chosen, lying on the line connecting the Asteroid with the points $A$ and $G$ respectively, at a distance of 2.5 and 2.3 times their distance from the Asteroid. These artificial equilibria can be generated with a thrust lower then $4 \times 10^{-4} N$ (still well within the feasible range), kept constant during the motion. The $x / y$ projection of the resulting orbits, for $\sim 3$ revolution of Jupiter around the Sun, is shown in Figure $18 \mathrm{a}$ ) and b). It must be noted that, due to the $x / y$ projection, the second orbit seems to spiral around the Asteroid but instead is displaced spatially. 

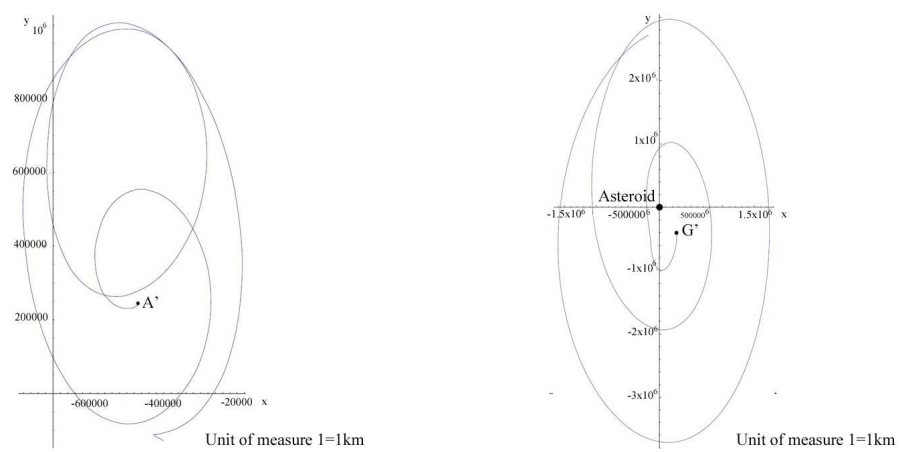

Fig. 18 Two orbits of the finite-time bounded zone of the real case after 3 Jovian years; a) The starting point $A^{\prime}$ is set on the line connecting $A$ and Hektor at a distance of $544903 \mathrm{~km}$ from the Asteroid; b) The starting point $G^{\prime}$ is set on the line connecting $G$ and Hektor at a distance of $711477 \mathrm{~km}$ from the Asteroid

Thus the simplified model studied here can give some indications of where weakly unstable solutions about a real Trojan may exist. Moreover it is clear that the perturbations due to a nonconstant velocity of revolution and the inclination of the tadpole orbits of the main Trojans must be taken into account when analysing stability.

\section{Summary}

A low thrust autonomous coplanar CRFBP, with the primaries set in the Lagrangian equilateral triangle configuration, has been formulated for both the purpose of mathematical interest as well as to investigate potential applications in the Sun-JupiterAsteroid-Spacecraft system. The analyses of the natural evolution of the system was performed, for a range of conceivable masses of a hypothetical asteroid set at the libration point $L_{4}$, which revealed eight natural equilibrium points, four of which are close to the asteroid. Of these, a linear stability analysis revealed that the two closest are unstable and the other two stable, when considering as primaries the Sun and two other bodies of the solar system.

Adding a low-thrust propulsion system to the spacecraft, which can thrust in every direction, including perpendicular to the plane containing the three massive bodies, a region of stable artificial equilibrium points close to the asteroid was created. As the "UK ion propulsion programme" has culminated in the availability of three thrusters of different sizes, covering the thrust $10^{-3} N$ to $0.3 N$ range (Fearn and Crookham, 2005), based on the $T 5, T 6$ and UK-25 propulsion systems, the assumed low-thrust potential for the spacecraft was initially based on current capability. Moreover completely novel, bounded orbits were proved to exist in the spatial vicinity of the asteroid which can be maintained with a constant thrust lower than the $0.1 \%$ of the maximum thrust capability, oriented in a fixed direction. Such result suggests that a shift towards micro propulsion systems (MPS) could be more appropriate for this application. Therefore, the Field Emission Electric Propulsion (FEEP) technology used in the Lisa pathfinder experiment on Einstein's geodesic motion in space, which operates in the micro-Newton field covering the range from $1 \times 10^{-7} N$ up to $5 \times 10^{-4} N$ (Nicolini, 2007), may provide a more effective propulsion system for the proposed application. 
This paper illustrates that by exploiting low-thrust, it would be possible to maintain strategic observation points, more than $66 \%$ closer to the hypothetical asteroid than the stable natural equilibrium points. This would enable a continuous synoptic view of the hypothetical asteroid itself.

A numerical simulation of the system was then performed, based on the real observations of the orbits of Jupiter and the 624-Hektor Trojan Asteroid to test the result when the asteroid is moving on its real tadpole orbit instead of remaining fixed in $L_{4}$. Results show a shift of the real model towards instability, i.e. the unstable zone enlarges, due to the inclusion in the real model of high perturbations.

However, numerical integration highlights a zone of finite-time bounded orbits for the real model, in which the orbits remain bounded within $\sim 3.5 \times 10^{6} \mathrm{~km}$ from the Asteroid for at least 36 Terrestrial years $(\sim 3$ Jupiter's and Hektor's revolutions around the Sun) before starting to escape. These finite-time bounded orbits can be maintained by using a constant thrust lower than $4 \times 10^{-4} N$ without the need for any state feedback control. 


\section{References}

Alvarez-Ramirez, M., Vidal, C.: 'Dynamical aspects of an equilateral restricted four-body prob- lem', pp. 1-23. Mathematical Problems in Engineering, Cambridge (2009)

Ambrosetti, A., Prodi, G.: 'A Primer of Nonlinear Analysis', pp. 153-159. Cambridge University Press, Cambridge (1993)

Andreu, M.A.,: 'New results on computation of translunar Halo orbits of the real EarthMoon system', Proceedings of the Conference Libration point orbits and applications, Aiguablava pp. 225-237 (2002)

Arnold, V.A., Kozolov, V.V, Neishtadt, A.I.: 'Mathematical aspects of classical and celestial mechanics. (Dynamical systems. III) ', Springer-Verlag, Berlin (2006)

Artin, M.: 'Algebra', pp. 543-547. Prentice Hall, New Jersey (1991)

Baig, S., McInnes, C.R.: 'Artificial Three Body Equilibria for Hybrid Low-Thrust Propulsion', J. Guid. Contr. Dynam., Vol. 31, No. 6, pp. 1644-1655 (2008)

Baig, S., McInnes, C.R.: 'Artificial halo orbits for low-thrust propulsion spacecraft', Celest. Mech. Dyn. Astr., Vol. 104, No. 4, pp. 321-335 (2009)

Baltagiannis, A.N., Papadakis K.E.: 'Equilibrium Points and Their Stability In The Restricted Four Body Problem', International Journal of Bifurcation and Chaos, World Scientific Publishing Company, accepted (2011)

Bombardelli C., Pelaez, J.: 'On the stability of artificial equilibrium points in the circular restricted three-body problem', Cele. Mech. and Dyn. Astr, Online First

Ceccaroni, M., Biggs, J.: 'Extension of low-thrust propulsion to the Autonomous Coplanar Circular Restricted Four Body Problem with application to future Trojan Asteroid missions', in $61^{\text {st }}$ International Astronautical Congress, IAC-10-1.1.3, Prague (2010)

Cronin, J., Richards, P.B., Russell, L.H.: 'Some periodic solutions of a four-body problem', Icarus, Vol. 3, p. 423 (1964)

Dvorak, R., Schwarz R., Lhotka Ch.: 'On the dynamics of Trojan planets in extrasolar planetary systems', International Astronomical Union (2008)

Erdi, B., Forgács-Dajka, E., Nagy, I., Rajnai, R.: 'A parametric study of stability and resonances around L4 in the elliptic restricted three-body problem', Celest. Mech. Dyn. Astr., Vol. 104, pp. 145-158 (2009)

Fearn, D.G., Crookham, C.: 'The development of ion propulsion in the UK: a historical prespective', Proceedings of the $29^{t h}$ IEPC, Princeton (2005)

Gascheau, M.: 'Examen d'une classe d'equations differentielles et application a un cas particulier du probleme des trois corps', Compt. Rend. 16, Princeton (1843)

Gomez, G., Jorba, A., Masdemont, J., Simo', C.: 'Dynamics and mission design near libration points vol.4: advanced methods for triangular points', World Scientific Monograph Series in Mathematics., Vol. 5, pp. 249-253 (2001)

Hadjidemetriou, J.D.: 'Instabilities in planetary-type orbits: applications to celestial mechanics', Instabilities in Dynamical Systems, Proceedings of the Advanced Study Institute, Cortina d'Ampezzo, pp. 135-163 (1978)

Hadjidemetriou, J.D.: 'On periodic orbits and resonance in extrasolar planetary systems', Celest. Mech. Dyn. Astr., Vol. 102, pp. 69-82 (2008)

Hadjidemetriou, J.D., Psychoyos, D., Voyatzis, G.: 'The 1/1 resonance in extrasolar planetary systems', Celest. Mech. Dyn. Astr., Vol. 104, pp. 23-38 (2011)

Hou, X.Y., Liu, L.: 'On quasi-periodic motions around the triangular libration points of the real EarthMoon system', Celest. Mech. Dyn. Astr., Vol. 108, No. 3, pp. 301-313 (2010) 
Kloppenborg, B., Stencel, R., Monnier, J.D., Schaefer, G., Zhao, M., Baron, F., McAlister, H., Brummelaar, T.T., Che, X., Farrington, C., Pedretti, E., Sallave-Goldfinger, P. J., Sturmann, J., Sturmann, L., Thureau, N., Turner, N., Carroll, S. M.: 'Infrared images of the transiting disk in the $\epsilon$ Aurigae system', pp.870-872., Nature Letters, 464, London (2010)

Koon, W.S., Lo, M., Marsden, J.E., Ross., S.: 'Dynamical Systems, the Three-Body Problem and Space Mission Design', pp. 123-130. Marsden Books, London (2008)

Marchal, C.,: 'Long term evolution of quasi-circular Trojan orbits', Celest. Mech. Dyn. Astr., Vol. 104, pp. 53-67 (2011)

Marzari F., Scholl, H., Murray, C., Lagerkvist, C.: 'Origin and Evolution of Trojan Asteroids', Asteroid III (2002)

Marzari F.: 'Puzzling Neptune Trojans', Science, Vol. 313, no. 5786, pp. 451-452 (2006)

McInnes, C.R., McDonald, A.J., John, F.L., MacDonald, E.W.: 'Solar sail parking in restricted three-body systems', J. Guid. Contr. Dynam., Vol. 17, No. 2, pp. 399-406 (1994)

McKay, R., Macdonald, M., Bosquillon de Frescheville, F., Vasile, M., McInnes, C.R., Biggs, J.: 'Non-Keplerian Orbits Using Low Thrust, High ISP Propulsion Systems', in $60^{\text {th }}$ International Astronautical Congress, IAC-09-1.2.8, Daejeon (2009)

McKay, R., Macdonald, M., Biggs, J., McInnes, C.R.: : 'Survey of Highly Non-Keplerian Orbits With Low-Thrust Propulsion', J. Guid. Contr. Dynam., Vol.34 no 3, pp. 645-666 (2011)

Melita, M.D., Licandro, J., Jones, D.C., Williams, I.P.: : 'Physical properties and orbital stability of the Trojan asteroids', Icarus, 195, pp. 686-697 (2008)

Michalodimitrakis, M.: 'The circular restricted four body problem', Astrophys. Space Sci., Vol. 75, No. 2, pp. 289-305 (1981)

Milani, A., Nobili, A.M.: 'On the stability of hierarchical four body systems', Celest. Mech. Dyn. Astr., Vol. 31, No. 3, pp. 241-291 (1983)

Morimoto, K., Yamakawa, M.Y., Uesugi, H.: 'Periodic Orbits with Low-Thrust Propulsion in the Restricted Three-Body Problem', J. Guid. Contr. Dynam., Vol. 29, No. 5, pp. 1131-1139 (2006)

Morimoto, K., Yamakawa, M.Y., Uesugi, H.: 'Artificial Equilibrium Points in the LowThrust Restricted Three-Body Problem', J. Guid. Contr. Dynam., Vol. 30, No. 5, pp. 1563-1568 (2007)

Multon F.R.: 'On a class of particular solutions of the problem of four bodies', Trans. of the American Math. Soc, 1, pp. 17-29 (1900)

Multon, F.R.: 'The straight line solutions of the problem of $\mathrm{N}$ bodies', Ann. of Math., Vol. 12, No. 3, pp. 1-17 (1910)

Nicolini, D.: 'LISA Pathfinder Field Emission Thruster System Development Program', Proceedings of the $30^{t h}$ International Electric Propulsion Conference, Florence, (2007)

Papadakis, K.E.: 'Asymptotic orbits in the restricted four body problem', Planet. Space Sci., Vol. 55, No. 10, pp. 1368-1379 (2007)

Piña, E., Lonngi, P.: 'Central configurations for the planar Newtonian four-body problem', Celest. Mech. Dyn. Astr., Vol. 108, No. 1, pp. 73 - 93 (2009)

Rivkin, A.S., Emery, J., Barucci, A., Bell, J.F., Bottke, W.F., Dotto, E., Gold, R., Lisse, C., Licandro, J., Prockter, L., Hibbits, C., Paul, M., Springmann, A., Yang, B.: 'The Trojan Asteroids: Keys to Many Locks', SBAG Community White Papers (2009)

Routh, E.J.: 'On Laplace's three particles, with a supplement on the stability of steady motion',Proc. Lond. Math. Soc., 6, pp. 86-97 (1875) 
Roy, A.E., Walker, I.W., MacDonald, A.J.C.: : 'Studies on the stability of hierarchical dynamical systems', Stability of the solar system and its minor natural and artificial bodies, Proceedings of the Advanced Study Institute, Cortina d'Ampezzo, pp. 151$174(1985)$

Scheeres, D.J.: 'The Restricted Hill Four-Body Problem with Applications to the Earth Moon Sun System', Celest. Mech. Dyn. Astr., Vol. 70, No. 2, pp. 75-98 (1998)

Schwarz, R., Suli, A., Dvorak, R. : 'Dynamics of possible Trojan planets in binary systems', Mon. Not. R. Astron. Soc., 398, pp. 2085-2090 (2009)

Schwarz, R., Süli, ., Dvorak,R., Pilat-Lohinger, E.: 'Stability of Trojan planets in multiplanetary systems Stability of Trojan planets in different dynamical systems', Celest. Mech. Dyn. Astr., Vol. 104, num.1-2, pp. 69-84 (2009)

Shoemaker, E.M., Shoemaker, C.S., Wolfe, R.F.: 'Trojan asteroids: populations, dynamical structure and origin of the L4 and L5 swarms', Asteroids II, Proceedings of the Conference, Tucson (1988)

Sicardy, B.: 'Stability of the triangular Lagrange points beyond Gascheaus value', Celest. Mech. Dyn. Astr., Vol. 107, No. 1-2, pp. 145-155 (2010)

Simò, C.: 'Relative equilibrium solutions in the four body problem', Celest. Mech. Dyn. Astr., Vol. 18, No. 2, pp. 165-184 (1978)

Steves, B.A., Roy, A.E., Bell, M.: 'Some special restricted four-body problems I. Modelling the Caledonian problem', Planet. Space Sci., Vol. 46, No. 11-12, pp. 1465-1474 (1998)

Steves, B.A., Roy, A.E., Bell, M.: 'Some special solutions of the four body problem - II. From Caledonia to Copenaghen', Planet. Space Sci., Vol. 46, No. 11-12, pp. 14751486 (1998)

Van Hamme, W., Wilson, R.E.: 'The restricted four-body problem and epsilon Aurigae',The Astroph. J., 306, pp. 33-36 (1986)

Waters, T., McInnes, C.R.: 'Periodic orbits above the ecliptic plane in the solar sail restricted 3-body problem', J. Guid. Contr. Dynam., Vol. 30, No. 3, pp. 687-693 (2007) 


\section{Appendix A}

We are interested in the equilibrium points $M_{j}, j=1, \ldots, 8$ defined at the three intersect of the surfaces that satisfy the equations of (2). As the third equation (i.e. $\left.\frac{\partial \Omega}{\partial z}=0\right)$ is clearly satisfied by the plane $z=0$, the equilibrium points $M_{j}, \quad j=1, \ldots, 8$, are bounded to stay on the $z=0$ plane, which, equivalently, can be seen as the degeneration of system (2) into a two dimensional system, once the solution of the third equation, namely $z=0$, is substituted in. Such system is qualitatively the same treated in Ceccaroni and Biggs (2010) whose solution is shown by Figure 19. In particular the light curve in the figure represents the solution of the first equation of system (2), the dark line shows the solution of the second equation of the system and the third equation (i.e. $\frac{\partial \Omega}{\partial z}=0$ ) is clearly satisfied by the plane $z=0$, plotted in all the Figure as well.

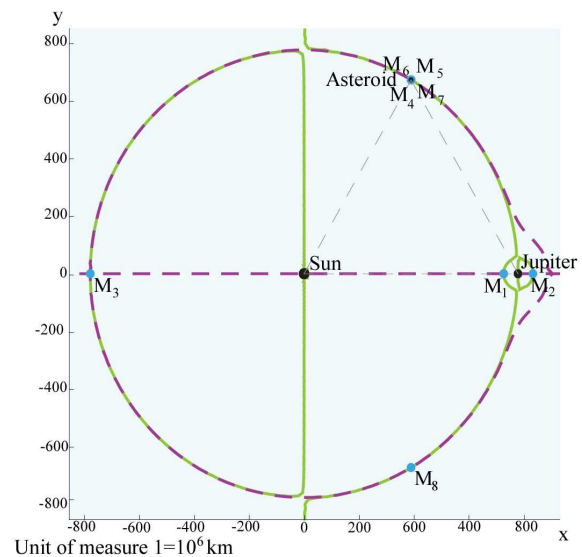

Fig. 19 Equilibrium points; Intersection with the plane $z=0$ 


\section{Appendix B}

Let be $\lambda, \lambda^{*} ; \nu, \nu^{*} ; \varphi, \varphi^{*} \in \mathbb{C}$ the eigenvalues of the system (31) and $\mathbf{f}_{j}=\mathbf{u}_{j}+i \mathbf{w}_{j} \in$ $\mathbb{R}^{6}, \quad j=1, \ldots, 6$ the respective eigenvectors.

Since $\left\{\mathbf{u}_{1}, \mathbf{w}_{1}, \mathbf{u}_{3}, \mathbf{w}_{3}, \mathbf{u}_{5}, \mathbf{w}_{5}\right\}$ is a base of $\mathbb{R}^{6}$, then $\forall s \in \mathbb{R}^{6}$ there exist $s_{1}, \ldots, s_{6} \in$ $\mathbb{R}$ such that $s=s_{1} \mathbf{u}_{1}+s_{2} \mathbf{w}_{1}+s_{3} \mathbf{u}_{3}+s_{4} \mathbf{w}_{3}+s_{5} \mathbf{u}_{5}+s_{6} \mathbf{w}_{5}$.

$$
\mathcal{A}^{\prime}\left(\begin{array}{c}
s_{1} \\
s_{2} \\
s_{3} \\
s_{4} \\
s_{5} \\
s_{6}
\end{array}\right)=\ldots=s_{1}\left(\begin{array}{c}
\mathcal{A}_{1,1}^{\prime} \\
\mathcal{A}_{2,1}^{\prime} \\
\mathcal{A}_{3,1}^{\prime} \\
\mathcal{A}_{4,1}^{\prime} \\
\mathcal{A}_{5,1}^{\prime} \\
\mathcal{A}_{6,1}^{\prime}
\end{array}\right)+\ldots+s_{6}\left(\begin{array}{c}
\mathcal{A}_{1,6}^{\prime} \\
\mathcal{A}_{2,6}^{\prime} \\
\mathcal{A}_{3,6}^{\prime} \\
\mathcal{A}_{4,6}^{\prime} \\
\mathcal{A}_{5,6}^{\prime} \\
\mathcal{A}_{6,6}^{\prime}
\end{array}\right)
$$

Then, of course, being the $\mathcal{A}$ and $\mathcal{A}^{\prime}$ the expression of the same system in two different coordinates, and being $\mathcal{M}$ the matrix of the change of coordinates, yields that (44) is also equal to:

$$
\begin{aligned}
& \mathcal{A}\left(\begin{array}{l}
s_{1} M_{1,1}+s_{2} M_{2,1}+s_{3} M_{3,1}+s_{4} M_{4,1}+s_{5} M_{5,1}+s_{6} M_{6,1} \\
s_{1} M_{1,2}+s_{2} M_{2,2}+s_{3} M_{3,2}+s_{4} M_{4,2}+s_{5} M_{5,2}+s_{6} M_{6,2} \\
s_{1} M_{1,3}+s_{2} M_{2,3}+s_{3} M_{3,3}+s_{4} M_{4,3}+s_{5} M_{5,3}+s_{6} M_{6,3} \\
s_{1} M_{1,4}+s_{2} M_{2,4}+s_{3} M_{3,4}+s_{4} M_{4,4}+s_{5} M_{5,4}+s_{6} M_{6,4} \\
s_{1} M_{1,5}+s_{2} M_{2,5}+s_{3} M_{3,5}+s_{4} M_{4,5}+s_{5} M_{5,5}+s_{6} M_{6,5} \\
s_{1} M_{1,6}+s_{2} M_{2,6}+s_{3} M_{3,6}+s_{4} M_{4,6}+s_{5} M_{5,6}+s_{6} M_{6,6}
\end{array}\right) \\
& =s_{1} \mathcal{A}\left(\begin{array}{c}
M_{1,1} \\
M_{2,1} \\
M_{3,1} \\
M_{5,1} \\
M_{6,1}
\end{array}\right)+\ldots+s_{6} \mathcal{A}\left(\begin{array}{l}
M_{1,6} \\
M_{2,6} \\
M_{3,6} \\
M_{4,6} \\
M_{5,6} \\
M_{6,6}
\end{array}\right) \\
& =s_{1}\left(\begin{array}{l}
\mathcal{A}\left(\mathbf{u}_{1}\right) / \mathbf{u}_{1} \\
\mathcal{A}\left(\mathbf{u}_{1}\right) / \mathbf{w}_{1} \\
\mathcal{A}\left(\mathbf{u}_{1}\right) / \mathbf{u}_{3} \\
\mathcal{A}\left(\mathbf{u}_{1}\right) / \mathbf{w}_{3} \\
\mathcal{A}\left(\mathbf{u}_{1}\right) / \mathbf{u}_{5} \\
\mathcal{A}\left(\mathbf{u}_{1}\right) / \mathbf{w}_{5}
\end{array}\right)+\ldots+s_{6}\left(\begin{array}{l}
\mathcal{A}\left(\mathbf{w}_{5}\right) / \mathbf{u}_{1} \\
\mathcal{A}\left(\mathbf{w}_{5}\right) / \mathbf{w}_{1} \\
\mathcal{A}\left(\mathbf{w}_{5}\right) / \mathbf{u}_{3} \\
\mathcal{A}\left(\mathbf{w}_{5}\right) / \mathbf{w}_{3} \\
\mathcal{A}\left(\mathbf{w}_{5}\right) / \mathbf{u}_{5} \\
\mathcal{A}\left(\mathbf{w}_{5}\right) / \mathbf{w}_{5}
\end{array}\right)
\end{aligned}
$$

Where, with / it is indicated the projection, e.g. $\mathcal{A}\left(\mathbf{u}_{1}\right) / \mathbf{u}_{1}$ means the component of $\mathcal{A}\left(\mathbf{u}_{1}\right)$ in the $\mathbf{u}_{1}$ direction.

Equating (44) and (45) we find that, being $\mathcal{A}^{\prime}=\left(\mathcal{A}_{j, k}^{\prime}\right)_{\substack{j=1, \ldots, 6 \\ k=1, \ldots, 6}}$,

$$
\mathcal{A}_{j, k}^{\prime}=\left\{\begin{array}{l}
\mathcal{A}\left(\mathbf{u}_{k}\right) / \mathbf{u}_{j} \text { if } k, j \text { odd } \\
\mathcal{A}\left(\mathbf{w}_{k-1} / \mathbf{u}_{j} \text { if } k \text { even, } j\right. \text { odd } \\
\mathcal{A}\left(\mathbf{u}_{k}\right) / \mathbf{w}_{j-1} \text { if } j \text { even, } k \text { odd } \\
\mathcal{A}\left(\mathbf{w}_{k-1}\right) / \mathbf{w}_{j-1} \text { if } k, j \text { even }
\end{array}\right.
$$

Now, given $\mathcal{A} \in \mathcal{L}\left(\mathbb{R}^{6}\right)$, let us define $\mathcal{A}_{\mathbb{C}}$ as the "complexification" of $\mathcal{A}$, namely, $\mathcal{A}_{\mathbb{C}} \in \mathcal{L}\left(\mathbb{C}^{6}\right)$ such that $\forall \mathbf{f}=\mathbf{u}+i \mathbf{w} \in \mathbb{C}^{6}, \mathbf{u}, \mathbf{w} \in \mathbb{R}^{6}, \mathcal{A}_{\mathbb{C}}(\mathbf{f}):=\mathcal{A}(\mathbf{u})+i \mathcal{A}(\mathbf{w})$. 
Then, taking $\mathcal{A}$ as in (31) and the first eigenvector $\mathbf{f}_{1} \in \mathbb{C}^{6}$ yields:

$$
\mathcal{A}_{\mathbb{C}}\left(\mathbf{f}_{1}\right)=\mathcal{A}\left(\mathbf{u}_{1}\right)+i \mathcal{A}\left(\mathbf{w}_{1}\right)
$$

But, since $\mathbf{f}_{1}$ is an eigenvector of $\mathcal{A}$, it is also true that:

$$
\mathcal{A}_{\mathbb{C}}\left(\mathbf{f}_{1}\right)=\lambda \mathbf{f}_{1}=\left(\lambda_{R}+i \lambda_{I}\right)\left(\mathbf{u}_{1}+i \mathbf{w}_{1}\right)=\left(\lambda_{R} \mathbf{u}_{1}-\lambda_{I} \mathbf{w}_{1}\right)+i\left(\lambda_{I} \mathbf{u}_{1}+\lambda_{R} \mathbf{w}_{1}\right)
$$

Equaling the Real and Imaginary parts of (47) and (48) yields:

$$
\begin{aligned}
& \mathcal{A}\left(\mathbf{u}_{1}\right)=\lambda_{R} \mathbf{u}_{1}-\lambda_{I} \mathbf{w}_{1} \\
& \mathcal{A}\left(\mathbf{w}_{1}\right)=\lambda_{R} \mathbf{w}_{1}+\lambda_{I} \mathbf{u}_{1}
\end{aligned}
$$

Repeating the analogous procedure for the other eigenvectors we finally find that:

$$
\mathcal{A}^{\prime}=\left(\begin{array}{llllll}
\lambda_{R} & \lambda_{I} & 0 & 0 & 0 & 0 \\
-\lambda_{I} & \lambda_{R} & 0 & 0 & 0 & 0 \\
0 & 0 & \nu_{R} & \nu_{I} & 0 & 0 \\
0 & 0 & -\nu_{I} & \nu_{R} & 0 & 0 \\
0 & 0 & 0 & 0 & \varphi_{R} & \varphi_{I} \\
0 & 0 & 0 & 0 & -\varphi_{I} & \varphi_{R}
\end{array}\right)
$$

Than, in this basis, the system takes the form

$$
\left.\dot{\Xi}(t)=\mathcal{A}^{\prime}\right\rfloor(t)
$$

with $\beth\left(t_{0}\right)=\left[\xi_{1}^{0}(t), \xi_{2}^{0}(t), \psi_{1}^{0}(t), \psi_{2}^{0}(t), \zeta_{1}^{0}(t), \zeta_{2}^{0}(t)\right]^{T}$. This system is solved by

$$
\beth(t)=\beth\left(t_{0}\right) e^{\mathcal{A}^{\prime} t}
$$

For the well known property of the exponential of a matrix ${ }^{12}$ we find that if $\mathcal{A}^{\prime}$ is as in (50) than $\mathcal{S}=e^{\mathcal{A}^{\gamma} t}$ is a block matrix $(6 \times 6)$ such that:

$$
\mathcal{S}=e^{\mathcal{A}^{\prime} t}=\left(\begin{array}{lll}
\mathcal{S}_{1} & 0 & 0 \\
0 & \mathcal{S}_{2} & 0 \\
0 & 0 & \mathcal{S}_{3}
\end{array}\right)
$$

with

$$
\begin{aligned}
\mathcal{S}_{1} & =\left(\begin{array}{l}
e^{\lambda_{R} t} \cos \left(\lambda_{I} t\right)-e^{\lambda_{R} t} \sin \left(\lambda_{I} t\right) \\
e^{\lambda_{R} t} \cos \left(\nu_{I} t\right) e^{\lambda_{R} t} \sin \left(\lambda_{I} t\right)
\end{array}\right) \\
\mathcal{S}_{2} & =\left(\begin{array}{l}
e^{\nu_{R} t} \cos \left(\nu_{I} t\right)-e^{\nu_{R} t} \sin \left(\nu_{I} t\right) \\
e^{\nu_{R} t} \cos \left(\nu_{I} t\right) e^{\nu_{R} t} \sin \left(\nu_{I} t\right)
\end{array}\right) \\
\mathcal{S}_{3} & =\left(\begin{array}{l}
e^{\varphi_{R} t} \cos \left(\varphi_{I} t\right)-e^{\varphi_{R} t} \sin \left(\varphi_{I} t\right) \\
e^{\varphi_{R} t} \cos \left(\varphi_{I} t\right) e^{\varphi_{R} t} \sin \left(\varphi_{I} t\right)
\end{array}\right)
\end{aligned}
$$

that is exactly the one in (33).

Finally, for how the solution has been derived, it is clear that $\mathrm{x}(t)=\mathcal{M} \Xi(t) \square$.

21 This property is straight forward from direct calculations once the basis in which the matrix of the system $\mathcal{A}$ is diagonal (but with complex coefficients) is known. 